\title{
Geometric Power Control for Time-Switching Energy-Harvesting Two-User Interference Channel
}

\author{
Yongxu Zhu, Kai-Kit Wong, Yangyang Zhang, and Christos Masouros
}

\begin{abstract}
This paper studies the optimization of power control for the two-user interference channel in which the terminals are time-switched between the communication and energy-harvesting phases. The objective is to maximize the sum-rate, subject to the minimum data and harvested energy constraints at the receivers, assuming a fixed time-switching coefficient. Our key contribution is a geometric approach that analyzes the feasible region governed by the constraints, which gives rise to the optimal power control solution. We assume that perfect channel state information (CSI) is available at both transmitters to determine the solution.
\end{abstract}

Index Terms-Energy harvesting, Interference channel, Power control, Sum-rate maximization, SWIPT, Time switching.

\section{INTRODUCTION}

I N RECENT years, green communications has been a main research theme in wireless communications, with an ever increasing focus on energy efficiency [1]. While power saving continues to be important, many have turned their attention to study ways to extend the lifetime of mobile terminals to realize genuine sustainability. The concept of simultaneous wireless information and power transfer (SWIPT) has thus emerged as a refreshing idea to exploit the power-bearing characteristics of wireless signals for possible remote charging [2-4].

SWIPT has already opened up new opportunities and led to numerous interesting but challenging optimization problems, e.g., [5-13]. In [5], an energy-harvesting sensor network was investigated, in which a sensor node uses the harvested energy from the environment to generate and transmit data packets. Energy management policies that are throughput-optimal and delay-minimal were devised. Subsequently in [6], the authors considered the use of energy harvesters as the energy sources and addressed the energy allocation problem according to the channel conditions and energy sources for maximizing the rate. Both causal and acausal side information were considered, and the optimal energy allocation was obtained using dynamic programming and convex optimization techniques.

Also, robust beamforming was addressed in [7] for SWIPT under the assumption that the channel state information (CSI) at the transmitter side was imperfect, with the errors confined within a worst-case deterministic model. Semidefinite relaxation (SDR) techniques were adopted to determine the optimal beamforming for maximizing the worst-case harvested energy

Copyright (c) 2015 IEEE. Personal use of this material is permitted. However, permission to use this material for any other purposes must be obtained from the IEEE by sending a request to pubs-permissions@ieee.org.

Zhu, Wong and Masouros are with the Department of Electronic and Electrical Engineering, University College London, Torrington Place, London, United Kingdom (e-mail: \{yongxu.zhu.13, kai-kit.wong\}@ucl.ac.uk).

Y. Zhang is with Kuang-Chi Institute of Advanced Technology, China. for the energy receiver (ER) while guaranteeing a target rate for the information receiver (IR). It was further shown that the relaxation is tight and as such, the robust-optimal beamforming solution for SWIPT can be efficiently obtained.

Recently, [8] considered the wireless link where the receiver switches between being an ER and an IR. When the receiver operates as an ER, it replenishes energy opportunistically from the unintended interference and/or the intended signal sent by the transmitter. To characterize the system performance tradeoffs, the outage-energy and rate-energy regions were analyzed. Given the CSI at the transmitter, the joint optimization of transmit power control, information and energy transfer scheduling, and the receiver's mode switching was also studied based on the instantaneous CSI and interference conditions.

Due to the broadcast nature of wireless channels, in SWIPT systems, the messages sent to IRs can be eavesdropped by the ERs. In [9], a multiuser multiple-input single-output (MISO) SWIPT system was considered and the maximization of the secrecy rate for the IR subject to individual harvested energy constraints of the ERs was studied. The problem of maximizing the weighted sum-energy at the ERs subject to a secrecy rate constraint for the IR was also investigated.

Recently, SWIPT has been considered for even more complex scenarios, e.g., [10-13]. In [10], Khandaker et al. studied the optimization of MISO beamforming for SWIPT employing power splitters (PSs) at the receivers in multicast communications channels. Using PS, each receiver can be an ER as well as an IR at the same time. The optimization problem of joint multicast transmit beamforming and receiving PS coefficients was addressed using SDR techniques. Later in [11], a secrecy problem similar to [9] was tackled, but with consideration of imperfect CSI. The problem was further investigated in [12] to cope with the case that the ERs can collude to perform joint decoding to illicitly decode the secret message to the IR.

Compared to the PS approach, SWIPT can be realized using time-switching at more affordable complexity. Very recently in [13], the authors provided a thorough study for time-switching SWIPT in multiple-input multiple-output (MIMO) interference channels. Assuming that perfect cancelation of energy signals is possible at the receivers, collaborative energy beamforming was obtained. Further, [13] studied the achievable rate-energy regions for the four possible modes of ER/IR for the two-user case. For more users, pairwise cooperation was proposed.

For complexity sake, time-switching SWIPT appears more appealing than the PS approach. Though [13] provided a useful study for time-switching SWIPT, there are further important problems that need investigating. Firstly, collaborative energy beams may not always be possible in the interference channel. 


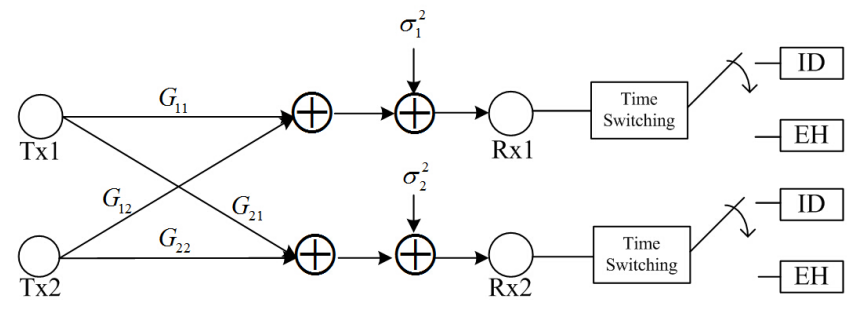

Fig. 1. A time-switching SWIPT network model.

Secondly, in the interference channel, meeting the target rates at individual IRs can sometimes be much more important than maximizing their sum-rate (the latter being the focus of [13]). Most recently in [14], a block-based time-switching protocol was proposed for powering relaying transmission.

Motivated by this, in this paper, we investigate the two-user interference channel where both users operate synchronously in fixed time-switching manner, for wireless information transfer (WIT) and wireless power transfer (WPT). Both users are subject to individual data and energy harvesting requirements, while aiming for maximizing their channel sum-rate. Assuming a fixed prescribed time-switching factor, our problem of interest is to find the optimal power control for both users to achieve this, given perfect channel state information (CSI). In particular, we address this non-convex optimization problem by analyzing the geometry of the feasible region.

In particular, our work differs from [13] in that the sum-rate maximization is studied with both rate and energy harvesting constraints whereas [13] did not have rate or energy harvesting constraints in the optimization. The inclusion of the rate and energy harvesting constraints is what makes our optimization of the users' transmit power so much more difficult compared to the ones in [13]. On the other hand, [14] studied optimization of the time-switching parameter $\tau$ for a relaying channel with WPT. Clearly, the inter-user interference aspect and the trade-off between WIT and WPT in the interference-limited environment that our work has tackled were not addressed.

The remainder of this paper is organized as follows. Section II introduces the network model. In Section III, we first present some lemmas which are key to understanding the geometric properties of the constraints for maximizing the sum-rate, and then present the optimal power allocation for the cases of rate constraints only, energy constraints only, and both data and energy constraints. Section IV provides the numerical results and we finally conclude the paper in Section V.

\section{Two-User TIME-Switching SWIPT}

We consider a two-user SWIPT communications network in which two transmitters communicate with their own receivers on a single radio channel over flat fading. Hence, they interfere with each other. As shown in Fig. 1, the considered SWIPT system operates in time switching fashion, switching between IR and ER, with $\tau$ units of time dedicated for WPT while the remaining $T-\tau$ units of time for WIT. The time-switching factor, $\tau$, is assumed fixed and adopted by both users.

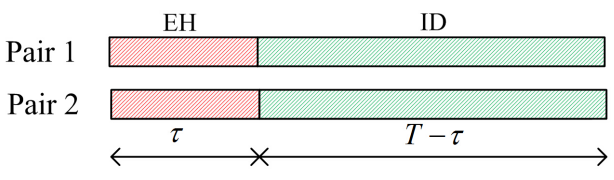

Fig. 2. Illustration of the time switching operation.

This is a crosstalk interference channel which is characterized by the two-input two-output channel gain matrix

$$
\mathbf{G}=\left[\begin{array}{ll}
G_{1,1} & G_{1,2} \\
G_{2,1} & G_{2,2}
\end{array}\right] .
$$

During the WIT phase, both users' receivers act as IRs, and the signal-to-interference plus noise ratio (SINR) at the $n$th receiver can be written as

$$
\operatorname{SINR}_{n}=\frac{P_{n}^{i} G_{n, n}}{\sigma_{n}^{2}+P_{m}^{i} G_{n, m}}, \text { for } m \neq n, \text { and } m, n=1,2,
$$

where $P_{1}^{i}$ and $P_{2}^{i}$ denote, respectively, the transmit power for user 1 and user 2 in the WIT phase, and $\sigma_{n}^{2}$ denotes the noise power at receiver $n$. To maximize the utilization of the channel, it is customary to maximize the achievable sum-rate

$$
\begin{aligned}
R_{\text {sum }} & \equiv(T-\tau)\left(R_{1}+R_{2}\right), \\
& =(T-\tau)\left(\log _{2}\left(1+\operatorname{SINR}_{1}\right)+\log _{2}\left(1+\operatorname{SINR}_{2}\right)\right) .
\end{aligned}
$$

Therefore, we have the maximization problem:

$$
\max _{\boldsymbol{P}^{i}} \log _{2}\left(1+\operatorname{SINR}_{1}\left(\boldsymbol{P}^{i}\right)\right)+\log _{2}\left(1+\operatorname{SINR}_{2}\left(\boldsymbol{P}^{i}\right)\right),
$$

where $\boldsymbol{P}^{i} \triangleq\left(P_{1}^{i}, P_{2}^{i}\right)$, and the factor $(T-\tau)$ is removed since $T$ and $\tau$ are constants and do not affect the optimization.

For WPT, all received powers (including the noise power) contribute to the harvested power. In particular, without loss of generality, assuming 100\% harvesting efficiency, the harvested power at the receivers is, respectively, given by

$$
\begin{aligned}
\frac{\mathcal{W}_{n}}{\tau}=Y_{n}=P_{n}^{e} G_{n, n}+ & \sigma_{n}^{2}+P_{m}^{e} G_{n, m}, \\
& \text { for } m \neq n, \text { and } m, n=1,2,
\end{aligned}
$$

where $\mathcal{W}_{n}$ denotes the energy received in the entire WPT slot, and hence $Y_{n}$ represents the received power, and $P_{n}^{e}$ denotes the transmit power for user $n$ in the WPT phase.

For the two-user interference channel, it is important to meet individual constraints and in SWIPT, it is considered that each user should be given a data constraint for WIT and a harvested energy constraint for WPT. Mathematically, we have

$$
\begin{aligned}
Y_{1} \tau & \geq \underline{\mathcal{W}}_{1}, \\
Y_{2} \tau & \geq \underline{\mathcal{W}}_{2}, \\
R_{1}(T-\tau) & \geq \underline{\mathcal{D}}_{1}, \\
R_{2}(T-\tau) & \geq \underline{\mathcal{D}}_{2},
\end{aligned}
$$

where $\underline{\mathcal{W}}_{1}$ and $\underline{\mathcal{W}}_{2}$ are the minimum target harvested energy for user 1 and user 2 , respectively, and $\underline{\mathcal{D}}_{1}$ and $\underline{\mathcal{D}}_{2}$ denote the minimum target data for user 1 and user 2, respectively. 
As a result, the optimization problem for the fixed timeswitching SWIPT interference system over one complete WITWPT period can be written as

$$
\begin{gathered}
\max _{\boldsymbol{P}^{i}, \boldsymbol{P}^{e}} \quad R_{\text {sum }}=R_{1}+R_{2} \\
\text { s.t. }\left\{\begin{array}{c}
Y_{1} \geq \frac{\underline{\mathcal{W}}_{1}}{\tau}, \\
Y_{2} \geq \frac{\underline{\mathcal{W}}_{2}}{\tau}, \\
R_{1} \geq \frac{\underline{\mathcal{D}_{1}}}{T-\tau}, \\
R_{2} \geq \frac{\underline{\mathcal{D}_{2}}}{T-\tau}, \\
0 \leq P_{1}^{i}+P_{1}^{e} \leq \bar{P}_{1}, \\
0 \leq P_{2}^{i}+P_{2}^{e} \leq \bar{P}_{2} .
\end{array}\right.
\end{gathered}
$$

where $\boldsymbol{P}^{e} \triangleq\left(P_{1}^{e}, P_{2}^{e}\right)$, and $\bar{P}_{1}$ and $\bar{P}_{2}$ denote the maximum permissible power for transmitter 1 and transmitter 2 , respectively. Note that the peak power constraint is shared over the WIT and WPT phases. It is possible that the sum-rate can be improved if different power is allocated for the two phases. As $Y_{1}$ and $Y_{2}$ are increasing functions of $P_{1}^{e}$ and $P_{2}^{e}$, respectively, if $P_{1}^{i}$ and $P_{2}^{i}$ are decided, then we can set $P_{1}^{e}=\bar{P}_{1}-P_{1}^{i}$ and $P_{2}^{e}=\bar{P}_{2}-P_{2}^{i}$, without affecting the optimality and feasibility of (10). Therefore, we can rewrite (10) as

$$
\begin{aligned}
& \max _{P^{i}, \boldsymbol{P}^{e}} R_{\text {sum }}=R_{1}+R_{2} \\
& \text { s.t. }\left\{\begin{aligned}
Y_{1} & \geq \frac{\underline{\mathcal{W}}_{1}}{\tau}, \\
Y_{2} & \geq \frac{\mathcal{W}_{2}}{\tau}, \\
R_{1} & \geq \frac{\underline{\mathcal{D}}_{1}}{T-\tau}, \\
R_{2} & \geq \frac{\underline{\mathcal{D}}_{2}}{T-\tau}, \\
0 & \leq P_{1}^{i} \leq \bar{P}_{1}, \\
0 & \leq P_{2}^{i} \leq \bar{P}_{2}, \\
P_{1}^{e} & =\bar{P}_{1}-P_{1}^{i}, \\
P_{2}^{e} & =\bar{P}_{2}-P_{2}^{i} .
\end{aligned}\right.
\end{aligned}
$$

\section{Optimal Power CONTRol}

This section uses a geometrical analysis to find the optimal power pair allocation for (11) in the transmit power region $\Pi=\left\{\left(P_{1}^{i}, P_{2}^{i}\right) \mid 0 \leq P_{1}^{i} \leq \bar{P}_{1}, 0 \leq P_{2}^{i} \leq \bar{P}_{2}\right\}$. As stated above, it suffices to obtain the optimal $\boldsymbol{P}^{i} \subseteq \Pi$, as $\boldsymbol{P}^{e}$ can be found from $\boldsymbol{P}^{i}$. To proceed, we have the following lemmas.

\section{A. Lemmas}

Lemma 1. In order to maximize the system sum-rate function $R_{\text {sum }}$, the optimal power allocation appears at either boundary of $P_{1}^{i}=\bar{P}_{1}^{i}$ or $P_{2}^{i}=\bar{P}_{2}^{i}$, where $\bar{P}_{k}^{i}$ denotes the maximum permissible transmit power for $P_{k}^{i}$ which is usually less than $\bar{P}_{k}$ because of meeting the energy harvesting constraints.

Proof: Since the log function is a monotonically increasing function, it suffices to look at the function

$$
f\left(\boldsymbol{P}^{i}\right) \triangleq\left(1+\frac{P_{1}^{i} G_{1,1}}{P_{2}^{i} G_{1,2}+\sigma_{1}^{2}}\right)\left(1+\frac{P_{2}^{i} G_{2,2}}{P_{1}^{i} G_{2,1}+\sigma_{2}^{2}}\right) .
$$

Given that $0 \leq P_{1}^{i} \leq \bar{P}_{1}^{i}$ and $0 \leq P_{2}^{i} \leq \bar{P}_{2}^{i}$, we proceed to analyze the maximization of $f$ with respect to (w.r.t.) $\boldsymbol{P}^{i}$.

Now, treating $P_{2}^{i}$ as fixed, differentiate $f$ w.r.t. $P_{1}^{i}$ to give

$$
\begin{aligned}
\frac{\partial f\left(\boldsymbol{P}^{i}\right)}{\partial P_{1}^{i}}= & \left(\frac{G_{1,1}}{A\left(P_{2}^{i}\right)}\right)\left(1+\frac{P_{2}^{i} G_{2,2}}{B\left(P_{1}^{i}\right)}\right) \\
& -\left(\frac{G_{2,1}}{B\left(P_{1}^{i}\right)}\right)\left(\frac{P_{2}^{i} G_{2,2}}{B\left(P_{1}^{i}\right)}\right)\left(1+\frac{P_{1}^{i} G_{1,1}}{A\left(P_{2}^{i}\right)}\right),
\end{aligned}
$$

where, for convenience, we have defined

$$
\left\{\begin{array}{l}
A\left(P_{2}^{i}\right)=P_{2}^{i} G_{1,2}+\sigma_{1}^{2}>0, \\
B\left(P_{1}^{i}\right)=P_{1}^{i} G_{2,1}+\sigma_{2}^{2}>0 .
\end{array}\right.
$$

If we have either $\frac{\partial f\left(\boldsymbol{P}^{i}\right)}{\partial P_{1}^{i}}>0$ or $\frac{\partial f\left(\boldsymbol{P}^{i}\right)}{\partial P_{1}^{i}}<0$, then $f\left(\boldsymbol{P}^{i}\right)$ is a monotonic function, and the maximum of $f\left(\boldsymbol{P}^{i}\right)$ will appear at an endpoint of $P_{1}^{i}$, i.e., either 0 or $\bar{P}_{1}^{i}$. On the other hand, if it is possible that $\frac{\partial f\left(\boldsymbol{P}^{i}\right)}{\partial P_{1}^{i}}=0$, then the maximum may occur at the $P_{1}^{i}$ such that $\frac{\partial f\left(\boldsymbol{P}^{i}\right)}{\partial P_{1}^{i}}=0$. To analyze this case, it can be easily shown that if $\frac{\partial f\left(\boldsymbol{P}^{i}\right)}{\partial P_{1}^{i}}=0$, then we have

$$
\frac{G_{2,1}}{B\left(P_{1}^{i}\right)}\left(1+\frac{P_{1}^{i} G_{1,1}}{A\left(P_{2}^{i}\right)}\right)-\frac{G_{1,1}}{A\left(P_{2}^{i}\right)}=\frac{G_{1,1} B\left(P_{1}^{i}\right)}{A\left(P_{2}^{i}\right) P_{2}^{i} G_{2,2}} .
$$

To find out whether such $P_{1}^{i}$ corresponds to a maximum or minimum, we obtain the second derivative $\frac{\partial^{2} f\left(\boldsymbol{P}^{i}\right)}{\partial P_{1}^{i 2}}$ as

$$
\begin{aligned}
& \frac{\partial^{2} f\left(P_{1}^{i}, P_{2}^{i}\right)}{\partial P_{1}^{i^{2}}}= \\
& \frac{2 P_{2}^{i} G_{2,2} G_{2,1}}{B^{2}\left(P_{1}^{i}\right)}\left[\frac{G_{2,1}}{B\left(P_{1}^{i}\right)}\left(1+\frac{P_{1}^{i} G_{1,1}}{A\left(P_{2}^{i}\right)}\right)-\frac{G_{1,1}}{A\left(P_{2}^{i}\right)}\right] .
\end{aligned}
$$

Using (15) on the above, it is found that at the turning point $\frac{\partial f\left(\boldsymbol{P}^{i}\right)}{\partial P_{1}^{i}}=0$, we have

$$
\frac{\partial^{2} f\left(\boldsymbol{P}^{i}\right)}{\partial P_{1}^{i^{2}}}=\frac{2 G_{2,2} G_{1,1}}{A\left(P_{2}^{i}\right) B\left(P_{1}\right)}>0,
$$

which means that it is a minimum and $f\left(\boldsymbol{P}^{i}\right)$ is convex, so the maximum occurs at an endpoint of $P_{1}^{i}$. A similar result for $P_{2}^{i}$ is also anticipated. Thus, we complete the proof.

Corollary 1. The power allocation pair $\boldsymbol{P}^{i}$ that maximizes the sum-rate is in the set of the corner points:

$$
\boldsymbol{P}^{i} \in\left\{\left(\bar{P}_{1}^{i}, 0\right),\left(0, \bar{P}_{2}^{i}\right),\left(\bar{P}_{1}^{i}, \bar{P}_{2}^{i}\right)\right\} .
$$

Proof: This is a direct result of Lemma 1.

Lemma 2. With the rate constraints, the feasible region for $\boldsymbol{P}^{i}$ is characterized by the half planes defined by the two straight lines on the $\left(P_{1}^{i}, P_{2}^{i}\right)$-plane, given by

$$
\begin{aligned}
& \boldsymbol{l}_{R_{1}}: P_{2}^{i}=\frac{1}{2^{\frac{\mathcal{D}_{1}}{T-\tau}}-1}\left(\frac{G_{1,1}}{G_{1,2}}\right) P_{1}^{i}-\frac{\sigma_{1}^{2}}{G_{1,2}}, \\
& \boldsymbol{l}_{R_{2}}: P_{2}^{i}=\left(2^{\frac{\mathcal{D}_{2}}{T-\tau}}-1\right)\left(\frac{G_{2,1}}{G_{2,2}}\right) P_{1}^{i}+\left(2^{\frac{\mathcal{D}_{2}}{T-\tau}}-1\right) \frac{\sigma_{2}^{2}}{G_{2,2}} .
\end{aligned}
$$

In particular, the feasible region is the intersection of the lower half plane of $\boldsymbol{l}_{R_{1}}$ and the upper half plane of $\boldsymbol{l}_{R_{2}}$. 
TABLE I

ATTRIBUTES OF THE RATE CONSTRAINT LINES.

\begin{tabular}{r||c|c} 
& $\boldsymbol{l}_{R_{1}}$ & $\boldsymbol{l}_{R_{2}}$ \\
\hline Slope & $\frac{1}{2^{\frac{\mathcal{D}}{T-\tau}}-1}\left(\frac{G_{1,1}}{G_{1,2}}\right)$ & $\left(2^{\frac{\mathcal{D}_{2}}{T-\tau}}-1\right)\left(\frac{G_{2,1}}{G_{2,2}}\right)$ \\
\hline$P_{1}^{i}$-intercept & $\left(2^{\frac{\mathcal{D}_{1}}{T-\tau}}-1\right) \frac{\sigma_{1}^{2}}{G_{1,1}}$ & $-\frac{\sigma_{2}^{2}}{G_{2,1}}$ \\
$\left.P_{1}^{i}\right|_{\left(\boldsymbol{l}_{R}, P_{2}^{i}=0\right)}$ & $-\frac{\sigma_{1}^{2}}{G_{1,2}}$ & $\left(2^{\frac{\mathcal{D}_{2}}{T-\tau}}-1\right) \frac{\sigma_{2}^{2}}{G_{2,2}}$ \\
\hline$P_{2}^{i}$-intercept & $\left.P_{2}^{i}\right|_{\left(\boldsymbol{l}_{R}, P_{1}^{i}=0\right)}$ &
\end{tabular}

$\S$ Note that throughout this paper, the notation $\left.P\right|_{\left(\boldsymbol{l}_{X}, l_{Y}\right)}$ is used to specify the value for $P$ at the intersection point of line $\boldsymbol{l}_{X}$ and line $\boldsymbol{l}_{Y}$.

Proof: The results can be immediately obtained by manipulating the rate (or data) constraints in (10). The attributes of the straight lines are summarized in Table I. Also, we can see that the slopes of both lines are positive; hence the angles enclosed by the straight lines and the $P_{1}^{i}$-axis are acute.

Corollary 2. The slope of $\boldsymbol{l}_{R_{1}}$ must be greater than the slope of $\boldsymbol{l}_{R_{2}}$, if problem (11) is feasible.

Proof: If the slope of $\boldsymbol{l}_{R_{1}}$ is smaller than the slope of $\boldsymbol{l}_{R_{2}}$, then the intersection area will occur at the third quadrant of the $\left(P_{1}^{i}, P_{2}^{i}\right)$-plane, which means that the required power $\boldsymbol{P}^{i}$ needs to be negative. Therefore, the contrary must be true.

Lemma 3. The sum-rate, $R_{\text {sum }}$, increases along the line $\boldsymbol{l}_{R_{1}}$ (or $l_{R_{2}}$ ) of increasing the transmit power $P_{1}^{i}$ and $P_{2}^{i}$.

Proof: We will focus on the proof for the sum-rate along line $\boldsymbol{l}_{R_{1}}$ when $P_{1}^{i}$ increases. The proof for other results follow similarly. As we know, on line $\boldsymbol{l}_{R_{1}}, R_{1}=\frac{\mathcal{D}_{1}}{T-\tau}$ is constant, and therefore, $R_{\text {sum }}$ increases if $R_{2}$ increases. To see this is indeed the case, we can express $P_{2}^{i}$ in terms of $P_{1}^{i}$ using $l_{R_{1}}$ in (19), and differentiate $R_{2}$ w.r.t. $P_{1}^{i}$, which shows

$$
\begin{aligned}
& \frac{\partial R_{2}}{\partial P_{1}^{i}}= \\
& \frac{1}{\ln 2}\left(\frac{1}{\left(\frac{1}{2^{\frac{\mathcal{D}}{T-\tau}}-1}\left(\frac{G_{1,1}}{G_{1,2}}\right) P_{1}^{i}-\frac{\sigma_{1}^{2}}{G_{1,2}}\right) G_{2,2}+P_{1}^{i} G_{2,1}+\sigma_{2}^{2}}\right) \\
& \quad \times\left(\frac{\left(\frac{1}{2^{\frac{\mathcal{D}}{T-\tau}}-1}\left(\frac{G_{1,1}}{G_{1,2}}\right) P_{1}^{i}-\frac{\sigma_{1}^{2}}{G_{1,2}}\right) G_{2,2} G_{2,1}}{P_{1}^{i} G_{2,1}+\sigma_{2}^{2}}\right)>0 .
\end{aligned}
$$

This implies that $R_{2}$ (and hence $R_{\text {sum }}$ ) is monotonic increasing in $P_{1}^{i}$ and $P_{2}^{i}$ along $\boldsymbol{l}_{R_{1}}$, which completes the proof.

The results of Lemma 2 and Corollary 2 together provide the feasible region given by the rate constraints mathematically. Fig. 3 shows the possible feasible regions graphically. In this figure, we have used the corner point $\left(\bar{P}_{1}, \bar{P}_{2}\right)$ to characterize the possible cases in which the lines $\boldsymbol{l}_{R_{1}}$ and $\boldsymbol{l}_{R_{1}}$ may cut the box region $\Pi$. In particular, the achievable rates for $\left(\bar{P}_{1}, \bar{P}_{2}\right)$,

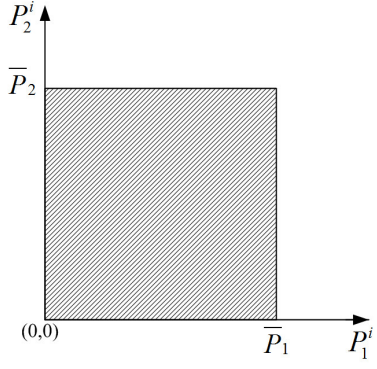

(a)

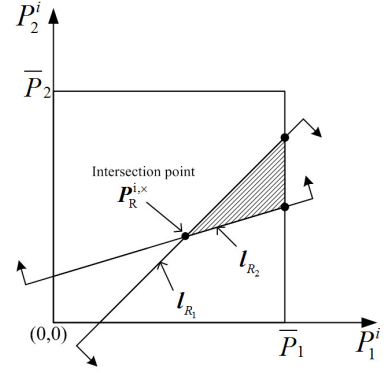

(c)

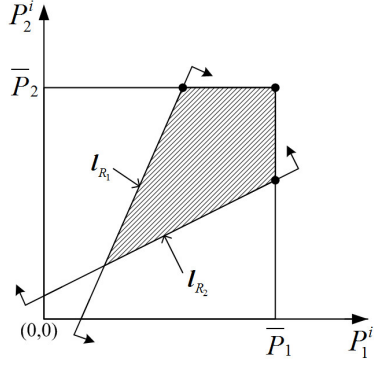

(b)

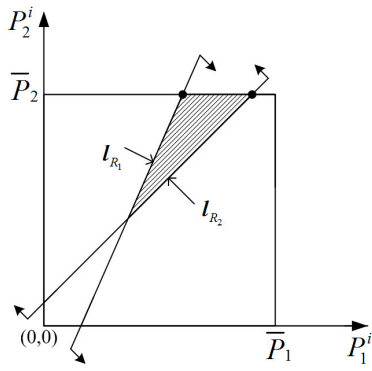

(d)
Fig. 3. Illustration of the possible feasible regions (shaded areas) for $\boldsymbol{P}^{i}$ when considering the rate constraints: (a) The box region $\Pi$, with only peak power constraints and no rate constraints; (b)-(d) $\Pi$ with peak power constraints and minimum rate constraints, (b) when $\underline{\mathcal{D}}_{1}<D_{1}^{\circ}$ and $\underline{D}_{2}<D_{2}^{\circ}$, (c) when $\mathcal{D}_{1}>D_{1}^{\circ}$ and $\mathcal{D}_{2}<D_{2}^{\circ}$, and (d) when $\underline{\mathcal{D}}_{1}<D_{1}^{\circ}$ and $\underline{D}_{2}>D_{2}^{\circ}$. Note that if $\underline{\mathcal{D}}_{1}>D_{1}^{\circ}$ and $\underline{\mathcal{D}}_{2}>D_{2}^{\circ}$, the intersection point will appear outside the box region $\Pi$ and in this case, no power will be feasible. For the same reason, due to Corollary 2, this figure only illustrates the cases if the slope of $\boldsymbol{l}_{R_{1}}>$ the slope of $\boldsymbol{l}_{R_{2}}$; otherwise, the intersection point will appear in the third quadrant of the $\left(P_{1}^{i}, P_{2}^{i}\right)$-plane and no power will be feasible.

denoted as $\left(D_{1}^{\circ}, D_{2}^{\circ}\right)$ are used as the references, i.e.,

$$
\begin{aligned}
& D_{1}^{\circ}=(T-\tau) \log _{2}\left(1+\frac{\bar{P}_{1} G_{1,1}}{\sigma_{1}^{2}+\bar{P}_{2} G_{1,2}}\right), \\
& D_{2}^{\circ}=(T-\tau) \log _{2}\left(1+\frac{\bar{P}_{2} G_{2,2}}{\sigma_{2}^{2}+\bar{P}_{1} G_{2,1}}\right) .
\end{aligned}
$$

For $\boldsymbol{l}_{R_{1}}$, the line divides the region into $R_{1}>\frac{\mathcal{D}_{1}}{T-\tau}$ (lower half plane) and $R_{1}<\frac{\mathcal{D}_{1}}{T-\tau}$ (upper half plane). For the case in Fig. 3(b), the rate achievable by user 1 at $\left(\bar{P}_{1}, \bar{P}_{2}\right)$ appears to be in the lower half plane of $\boldsymbol{l}_{R_{1}}$. Therefore, it corresponds to $D_{1}^{\circ}>\underline{D}_{1}$. Similar arguments for $\boldsymbol{l}_{R_{2}}$ will indicate that in this case, it also corresponds to the fact that $D_{2}^{\circ}>\underline{\mathcal{D}}_{2}$. Other cases in Fig. 3 can also be deduced in a similar way.

Alternatively, feasibility can be understood via the intersection point of $\boldsymbol{l}_{R_{1}}$ and $\boldsymbol{l}_{R_{2}}$, denoted as $\boldsymbol{P}_{\mathrm{R}}^{i, \times}=\left(P_{1, \mathrm{R}}^{i, \times}, P_{2, \mathrm{R}}^{i, \times}\right)$, which is given by

$$
\left\{\begin{array}{l}
P_{1, \mathrm{R}}^{i, \times}=\frac{\left(2^{\frac{\mathcal{D}_{1}}{T-\tau}}-1\right)\left(\left(2^{\frac{\mathcal{D}_{2}}{T-\tau}}-1\right) G_{1,2} \sigma_{2}^{2}+G_{2,2} \sigma_{1}^{2}\right)}{G_{1,1} G_{2,2}-\left(2^{\frac{\mathcal{D}_{1}}{T-\tau}}-1\right)\left(2^{\frac{\mathcal{D}_{2}}{T-\tau}}-1\right) G_{2,1} G_{1,2}}, \\
P_{2, \mathrm{R}}^{i, \times}=\frac{\left(2^{\frac{\mathcal{D}_{2}}{T-\tau}}-1\right)\left(G_{1,1} \sigma_{2}^{2}+\left(2^{\frac{\mathcal{D}_{1}}{T-\tau}}-1\right) G_{2,1} \sigma_{1}^{2}\right)}{G_{1,1} G_{2,2}-\left(2^{\frac{\mathcal{D}_{1}}{T-\tau}}-1\right)\left(2^{\frac{\mathcal{D}_{2}}{T-\tau}}-1\right) G_{2,1} G_{1,2}} .
\end{array}\right.
$$

Corollary 3. To be feasible with the data/rate constraints, the intersection point $\boldsymbol{P}_{\mathrm{R}}^{i, \times}$ must be inside the power region $\Pi$. 
Proof: This is a direct result of Lemma 2.

Lemma 4. For the energy harvesting constraints, the feasible region for our problem is characterized by the intersection of the lower half planes of the two straight lines on the $\left(P_{1}^{i}, P_{2}^{i}\right)$ plane that are, respectively, given by

$$
\left\{\begin{aligned}
l_{Y_{1}}: P_{2}^{i} & =\left(-\frac{G_{1,1}}{G_{1,2}}\right) P_{1}^{i} \\
& +\frac{1}{G_{1,2}}\left(\bar{P}_{1} G_{1,1}+\bar{P}_{2} G_{1,2}-\frac{\mathcal{W}_{1}}{\tau}+\sigma_{1}^{2}\right), \\
l_{Y_{2}}: P_{2}^{i} & =\left(-\frac{G_{2,1}}{G_{2,2}}\right) P_{1}^{i} \\
& +\frac{1}{G_{2,2}}\left(\bar{P}_{1} G_{2,1}+\bar{P}_{2} G_{2,2}-\frac{\mathcal{W}_{2}}{\tau}+\sigma_{2}^{2}\right) .
\end{aligned}\right.
$$

Proof: Using (5) with the substitution $P_{1}^{e}=\bar{P}_{1}-P_{1}^{i}$ and $P_{2}^{e}=\bar{P}_{2}-P_{2}^{i}$ in the energy harvesting constraints of (10) will result in the two straight lines (24). Moreover, both lines have negative slopes, which means that the angles enclosed by the lines and the $P_{1}^{i}$-axis are obtuse. As before, the attributes of the straight lines are provided in Table II.

In typical scenarios, we likely have the main channel gains greater than that of the crosstalk channels, i.e., $G_{1,1}>G_{1,2}$, and $G_{2,2}>G_{2,1}$. Therefore, we will have

$$
\text { slope of } l_{Y_{1}}=-\frac{G_{1,1}}{G_{1,2}}<-1
$$

and

$$
\text { slope of } \boldsymbol{l}_{Y_{2}}=-\frac{G_{2,1}}{G_{2,2}}>-1>\text { slope of } \boldsymbol{l}_{Y_{1}} \text {. }
$$

TABLE II

ATTRIBUTES OF THE ENERGY HARVESTING CONSTRAINT LINES.

\begin{tabular}{r||c|c} 
& $\boldsymbol{l}_{Y_{1}}$ & $\boldsymbol{l}_{Y_{2}}$ \\
\hline Slope & $-\frac{G_{1,1}}{G_{1,2}}$ & $-\frac{G_{2,1}}{G_{2,2}}$ \\
\hline$P_{1}^{i}$-intercept & $\frac{\bar{P}_{1} G_{1,1}+\bar{P}_{2} G_{1,2}-\frac{\mathcal{W}_{1}}{\tau}+\sigma_{1}^{2}}{G_{1,1}}$ & $\frac{\bar{P}_{1} G_{2,1}+\bar{P}_{2} G_{2,2}-\frac{\mathcal{W}_{2}}{\tau}+\sigma_{2}^{2}}{G_{2,1}}$ \\
$\left.P_{1}^{i}\right|_{\left(\boldsymbol{l}_{Y}, P_{2}^{i}=0\right)}$ & $P_{2}^{i}$-intercept & $\bar{P}_{1} G_{1,1}+\bar{P}_{2} G_{1,2}-\frac{\mathcal{W}_{1}}{\tau}+\sigma_{1}^{2}$ \\
$\left.P_{1,2}^{i}\right|_{\left(\boldsymbol{l}_{Y}, P_{1}^{i}=0\right)}$ & $\frac{\bar{P}_{1} G_{2,1}+\bar{P}_{2} G_{2,2}-\frac{\mathcal{W}_{2}}{\tau}+\sigma_{2}^{2}}{G_{2,2}}$ \\
\hline
\end{tabular}

Corollary 4. Typically, the $P_{1}^{i}$-intercepts and $P_{2}^{i}$-intercepts for both lines $\boldsymbol{l}_{Y_{1}}$ and $\boldsymbol{l}_{Y_{2}}$ are positive, and the energy harvesting constraints are activated.

Proof: As shown in Table II, for both $\boldsymbol{l}_{Y_{1}}$ and $\boldsymbol{l}_{Y_{2}}$, the $P_{1}^{i}-$ intercept and $P_{2}^{i}$-intercept have the same polarities. That is to say, they are either both positive or both negative. The feasible region for $\boldsymbol{P}^{i}$ is the intersection of the lower half planes made by $\boldsymbol{l}_{Y_{1}}$ and $\boldsymbol{l}_{Y_{2}}$ (according to Lemma 4 ), and the box region $\Pi$ due to the peak power constraints. If both of the intersects are negative, the intersection is null and the problem is infeasible, so the energy harvesting constraints are only meaningful when the $P_{1}^{i}$-intercept and $P_{2}^{i}$-intercept are both positive.

In terms of feasibility, the intersection point of $l_{Y_{1}}$ and $l_{Y_{2}}$,

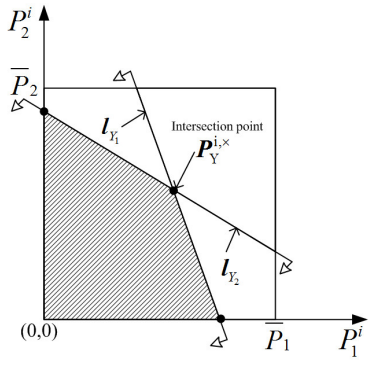

(a)

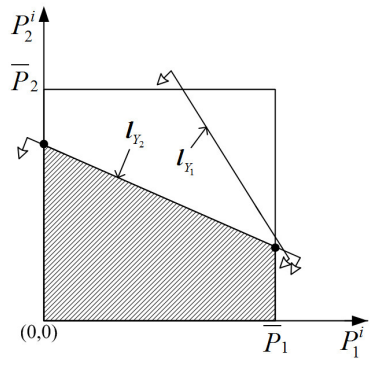

(b)

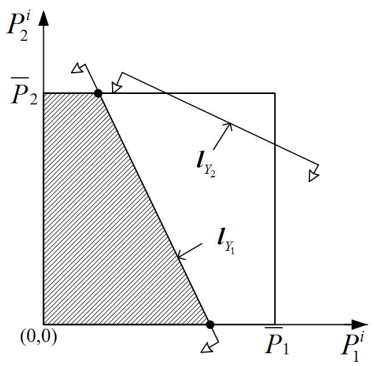

(c)

Fig. 4. Illustration of the possible feasible regions (shaded areas) for $\boldsymbol{P}^{i}$ when considering both the energy harvesting constraints and the peak power constraints $\Pi$, assuming the slope of $\boldsymbol{l}_{Y_{2}}>$ that of $\boldsymbol{l}_{Y_{1}}$ (the typical situation). In (a), $\boldsymbol{P}_{\mathrm{Y}}^{i, \times}$ occurs inside $\Pi$, or $0 \leq P_{1, \mathrm{Y}}^{i, \times} \leq \bar{P}_{1}$ and $0 \leq P_{2, \mathrm{Y}}^{i, \times} \leq \bar{P}_{2}$, while in (b), $\boldsymbol{P}_{\mathrm{Y}}^{i, \times}$ is outside $\Pi$ on the right, i.e., $P_{1, \mathrm{Y}}^{i, \times}>\bar{P}_{1}$ and $0 \leq P_{2, \mathrm{Y}}^{i, \times} \leq \bar{P}_{2}$. For (c), $\boldsymbol{P}_{\mathrm{Y}}^{i, \times}$ is above $\Pi$ or $0 \leq P_{1, \mathrm{Y}}^{i, \times} \leq \bar{P}_{1}$ and $P_{2, \mathrm{Y}}^{i, \times}>\bar{P}_{2}$. The case that the slope of $\boldsymbol{l}_{Y_{2}}<$ that of $\boldsymbol{l}_{Y_{1}}$ is also possible, and the analysis is similar. In the case that $\boldsymbol{P}_{\mathrm{Y}}^{i, \times}$ is far away from $\Pi$, the problem is infeasible.

denoted as $\boldsymbol{P}_{\mathrm{Y}}^{i, \times}=\left(P_{1, \mathrm{Y}}^{i, \times}, P_{2, \mathrm{Y}}^{i, \times}\right)$, which is given by

$$
\begin{aligned}
P_{1, \mathrm{Y}}^{i, \times} & =\frac{G_{2,2}\left(\bar{P}_{1} G_{2,1}+\bar{P}_{2} G_{2,2}-\frac{\underline{\mathcal{W}}_{2}}{\tau}+\sigma_{2}^{2}\right)}{G_{1,1} G_{2,2}-G_{1,2} G_{2,1}} \\
- & \frac{G_{2,1}\left(\bar{P}_{1} G_{1,1}+\bar{P}_{2} G_{1,2}-\frac{\underline{\mathcal{W}}_{1}}{\tau}+\sigma_{1}^{2}\right)}{G_{1,1} G_{2,2}-G_{1,2} G_{2,1}}, \\
P_{2, \mathrm{Y}}^{i, \times} & =\frac{G_{1,1}\left(\bar{P}_{1} G_{1,1}+\bar{P}_{2} G_{1,2}-\frac{\underline{\mathcal{W}}_{1}}{\tau}+\sigma_{1}^{2}\right)}{G_{1,1} G_{2,2}-G_{1,2} G_{2,1}} \\
- & \frac{G_{1,2}\left(\bar{P}_{1} G_{2,1}+\bar{P}_{2} G_{2,2}-\frac{\mathcal{W}_{2}}{\tau}+\sigma_{2}^{2}\right)}{G_{1,1} G_{2,2}-G_{1,2} G_{2,1}} .
\end{aligned}
$$

plays an important role. See caption of Fig. 4.

Fig. 4 depicts the possible feasible regions of $\boldsymbol{P}^{i}$ as far as the energy harvesting constraints are concerned.

Lemma 5. Along the energy harvesting constraint lines $\boldsymbol{l}_{Y_{1}}$ and $\boldsymbol{l}_{Y_{2}}$ specified in Lemma 4 , the maximum sum-rate, $R_{\text {sum }}$, occurs at the endpoints within the power constraints $\Pi$.

Proof: We will focus on line $l_{Y_{1}}$ but the proof follows naturally for $\boldsymbol{l}_{Y_{2}}$. On $\boldsymbol{l}_{Y_{1}}$, using (24), we can write $P_{2}^{i}$ in terms of $P_{1}^{i}$. Then as in the proof of Lemma 1, we substitute such 
$\boldsymbol{P}^{i}$ into $f$, and differentiate it w.r.t. $P_{1}^{i}$ to give

$$
\begin{gathered}
\frac{\partial f\left(\boldsymbol{P}^{i}\right)}{\partial P_{1}^{i}}=\frac{G_{1,1}}{M_{3}}\left(\frac{G_{2,2} M_{1}\left(P_{1}^{i}\right)}{M_{2}\left(P_{1}^{i}\right)}+1\right)\left(\frac{G_{1,1} P_{1}^{i}}{M_{3}}+1\right) \\
-\frac{G_{2,2}}{M_{2}\left(P_{1}^{i}\right)}\left(\frac{G_{2,1} M_{1}\left(P_{1}^{i}\right)}{M_{2}\left(P_{1}^{i}\right)}+\frac{G_{1,1}}{G_{1,2}}\right)\left(\frac{G_{1,1} P_{1}^{i}}{M_{3}}+1\right),
\end{gathered}
$$

where

$$
\begin{aligned}
& M_{1}=\frac{1}{G_{1,2}}\left(G_{1,1} \bar{P}_{1}+G_{1,2} \bar{P}_{2}-G_{1,1} P_{1}^{i}-\frac{\underline{\mathcal{W}}_{1}}{\tau}+\sigma_{2}^{2}\right)>0, \\
& M_{2}=\sigma_{2}^{2}+G_{2,1} P_{1}^{i}>0, \\
& M_{3}=\sigma_{1}^{2}+G_{1,2} M_{1}>0 .
\end{aligned}
$$

If $\frac{\partial f\left(\boldsymbol{P}^{i}\right)}{\partial P_{1}^{i}}$ is either always positive or always negative, then $f$ is monotonic and the result of this lemma follows. Otherwise, we need to show that $f$ is convex. To do so, we first obtain the condition for the turning point, $\frac{\partial f\left(\boldsymbol{P}^{i}\right)}{\partial P_{1}^{i}}=0$, giving

$$
\begin{aligned}
\frac{G_{1,1}^{2}}{M_{3}^{2}}\left(\frac{G_{2,2} M_{1}}{M_{2}}+1\right) & \\
& -\frac{G_{2,2} G_{1,1}}{M_{2} M_{3}}\left(\frac{G_{2,1} M_{1}}{M_{2}}+\frac{G_{1,1}}{G_{1,2}}\right)=0 .
\end{aligned}
$$

Next, we get the second-order derivative of $f$ at $\frac{\partial f\left(\boldsymbol{P}^{i}\right)}{\partial P_{1}^{i}}=0$ by deriving

$$
\begin{aligned}
\frac{\partial^{2} f\left(\boldsymbol{P}^{i}\right)}{\partial P_{1}^{i^{2}}}= & 2\left(\frac{G_{1,1} P_{1}}{M_{3}}+1\right)\left[\frac{G_{1,1}^{2}}{M_{2}^{2}}\left(\frac{G_{2,2} M_{1}}{M_{2}}+1\right)\right. \\
- & \frac{G_{2,2} G_{1,1}}{M_{2} M_{3}}\left(\frac{G_{2,1} M_{1}}{M_{2}}+\frac{G_{1,1}}{G_{1,2}}\right) \\
& \left.+\frac{G_{2,1} G_{2,2}}{M_{2}^{2}}\left(\frac{G_{2,1} M_{1}}{M_{2}}+\frac{G_{1,1}}{G_{1,2}}\right)\right] .
\end{aligned}
$$

Now, using (31) in the above, we can see that at the turning point, $\frac{\partial f\left(\boldsymbol{P}^{i}\right)}{\partial P_{1}^{i}}=0$, we have

$$
\begin{aligned}
& \frac{\partial^{2} f\left(\boldsymbol{P}^{i}\right)}{\partial P_{1}^{i^{2}}}= \\
& 2\left(\frac{G_{1,1} P_{1}}{M_{3}}+1\right) \frac{G_{2,1} G_{2,2}}{M_{2}^{2}}\left(\frac{G_{2,1} M_{1}}{M_{2}}+\frac{G_{1,1}}{G_{1,2}}\right)>0,
\end{aligned}
$$

which shows that $f$ (and hence the sum-rate) is convex over line $l_{Y_{1}}$. This concludes that the maximum sum-rate occurs at the endpoints of $l_{Y_{1}}$ over $\Pi$, which completes the proof.

\section{B. With Data/Rate Constraints Only}

Now, we derive the optimal power control according to the three possible scenarios in Fig. 3. According to Lemma 3, for the maximization of sum-rate, the optimal power control will appear at the edges of the box $\Pi$. In the following, we will give the optimal power control, $\boldsymbol{P}_{\mathrm{opt}}^{i}=\left(P_{1, \mathrm{opt}}^{i}, P_{2, \mathrm{opt}}^{i}\right)$, for maximizing the sum-rate with the rate constraints.
1) Scenario 1 (see Fig. 3(b)): In this case, the problem is feasible and $\boldsymbol{P}_{\mathrm{R}}^{i, \times}$ occurs within the permissible region $\Pi$. As shown in Lemma 3, the optimal $\boldsymbol{P}_{\mathrm{opt}}^{i}$ for maximizing the sumrate occurs at the edges of the box $\Pi, P_{1}^{i}=\bar{P}_{1}$ or $P_{2}^{i}=\bar{P}_{2}$. Because of the rate constraints, there are two intersections, one for $l_{R_{1}}$ and $P_{2}^{i}=\bar{P}_{2}$ in which case we denote

$$
P_{1}^{i}=\left.P_{1}^{i}\right|_{\left(\boldsymbol{l}_{R_{1}}, P_{2}^{i}=\bar{P}_{2}\right)}=\frac{\left(2^{\frac{\mathcal{D}_{1}}{T-\tau}}-1\right)}{G_{1,1}}\left(G_{1,2} \bar{P}_{2}+\sigma_{1}^{2}\right),
$$

and another one for $\boldsymbol{l}_{R_{2}}$ and $P_{1}^{i}=\bar{P}_{1}$ where

$$
P_{2}^{i}=\left.P_{2}^{i}\right|_{\left(l_{R_{2}}, P_{1}^{i}=\bar{P}_{1}\right)}=\frac{\left(2^{\frac{\mathcal{D}_{2}}{T-\tau}}-1\right)}{G_{2,2}}\left(G_{2,1} \bar{P}_{1}+\sigma_{2}^{2}\right) \text {. }
$$

Furthermore, from Corollary 1, we know that not only does the optimal $\boldsymbol{P}_{\mathrm{opt}}^{i}$ occur at the edges, it occurs at an endpoint of the feasible edges. In other words, we have

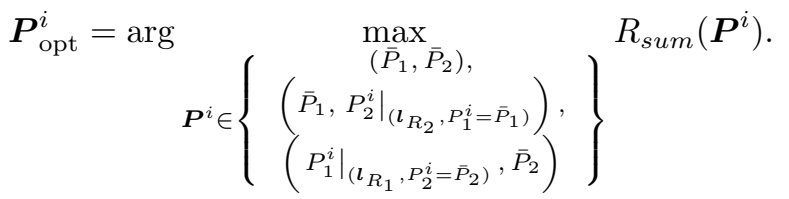

2) Scenario 2 (see Fig. 3(c)): As shown, in this case, both lines $\boldsymbol{l}_{R_{1}}$ and $\boldsymbol{l}_{R_{2}}$ intersect on $P_{1}^{i}=\bar{P}_{1}$. As such,

$$
\boldsymbol{P}_{\mathrm{opt}}^{i}=\arg \max ^{i \in\left\{\begin{array}{c}
\left(\bar{P}_{1},\left.P_{2}^{i}\right|_{\left(l_{R_{1}}, P_{1}^{i}=\bar{P}_{1}\right)}\right), \\
\left(\bar{P}_{1},\left.P_{2}^{i}\right|_{\left(l_{R_{2}}, P_{1}^{i}=\bar{P}_{1}\right)}\right)
\end{array}\right\}} R
$$

where

$$
\left.P_{2}^{i}\right|_{\left(\boldsymbol{l}_{R_{1}}, P_{1}^{i}=\bar{P}_{1}\right)}=\frac{1}{G_{1,2}}\left(\frac{G_{1,1} \bar{P}_{1}}{2^{\frac{\mathcal{D}_{1}}{T-\tau}}-1}-\sigma_{1}^{2}\right) .
$$

3) Scenario 3 (see Fig. 3(d)): Another possible situation is that both the lines $\boldsymbol{l}_{R_{1}}$ and $\boldsymbol{l}_{R_{2}}$ intersect on $P_{2}^{i}=\bar{P}_{2}$. As such, the optimal $\boldsymbol{P}_{\mathrm{opt}}^{i}$ can be found by

$$
\boldsymbol{P}_{\mathrm{opt}}^{i}=\arg \boldsymbol{P}^{i \in}\left\{\begin{array}{c}
\max \\
\left(\left.P_{1}^{i}\right|_{\left(l_{R_{1}}, P_{2}^{i}=\bar{P}_{2}\right)}, \bar{P}_{2}\right), \\
\left(\left.P_{1}^{i}\right|_{\left(l_{R_{2}}, P_{2}^{i}=\bar{P}_{2}\right)}, \bar{P}_{2}\right)
\end{array}\right\} R^{\text {sum }}\left(\boldsymbol{P}^{i}\right),
$$

where

$$
\left.P_{1}^{i}\right|_{\left(\boldsymbol{l}_{R_{2}}, P_{2}^{i}=\bar{P}_{2}\right)}=\frac{1}{G_{2,1}}\left(\frac{G_{2,2} \bar{P}_{2}}{2^{\frac{\mathcal{D}_{2}}{T-\tau}}-1}-\sigma_{2}^{2}\right) .
$$

\section{With Energy Harvesting Constraints Only}

The results in Lemma 5 and the intersection point $\boldsymbol{P}_{\mathrm{Y}}^{i, \times}$ will be useful in determining the optimal $\boldsymbol{P}_{\mathrm{opt}}^{i}$ with the energy harvesting constraints, which we detail below. 
1) Scenario 1 (see Fig. 4(a)): When the point of intersection $\boldsymbol{P}_{\mathrm{Y}}^{i, \times}$ is inside the box region $\Pi$, two points are of interest. According to Fig. 4(a), the first one is the intersection point between line $l_{Y_{1}}$ and line $P_{2}^{i}=0$, denoting as $\left(\left.P_{1}^{i}\right|_{\left(l_{Y_{1}}, P_{2}^{i}=0\right)}, 0\right)$, and another one is the intersection point between $t_{Y_{2}}$ and line $P_{1}^{i}=0$, or $\left(0,\left.P_{2}^{i}\right|_{\left(l_{Y_{2}}, P_{1}^{i}=0\right)}\right)$, where

$$
\left.P_{1}^{i}\right|_{\left(\boldsymbol{l}_{Y_{1}}, P_{2}^{i}=0\right)}=\frac{1}{G_{1,1}}\left(\bar{P}_{1} G_{1,1}+\bar{P}_{2} G_{1,2}-\frac{\underline{\mathcal{W}}_{1}}{\tau}+\sigma_{1}^{2}\right)
$$

and

$$
\left.P_{2}^{i}\right|_{\left(l_{Y_{2}}, P_{1}^{i}=0\right)}=\frac{1}{G_{2,2}}\left(\bar{P}_{1} G_{2,1}+\bar{P}_{2} G_{2,2}-\frac{\underline{\mathcal{W}}_{2}}{\tau}+\sigma_{2}^{2}\right) .
$$

Using Lemma 5, the optimal $\boldsymbol{P}_{\mathrm{opt}}^{i}$ can thus be found by

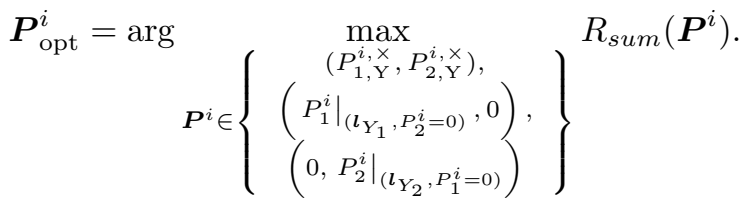

However, Fig. 4(a) illustrates only one of the many possibilities. In fact, $\boldsymbol{l}_{Y_{2}}$ may cut the top side of $\Pi$ instead of the left side. In this case, the point of interest will be the intersection point between $l_{Y_{2}}$ and line $P_{2}^{i}=\bar{P}_{2}$ but not the line $P_{1}^{i}=0$. Specifically, this will happen if $\left.P_{2}^{i}\right|_{\left(l_{Y_{2}}, P_{1}^{i}=0\right)}>\bar{P}_{2}$. Moreover, from Corollary 1 , the point $\left(0, \bar{P}_{2}\right)$ is now feasible and has a higher sum-rate than any other feasible point, and hence is the optimal power control solution. Similarly, $\boldsymbol{l}_{Y_{1}}$ may cut the right side of $\Pi$ instead of the bottom side, and we can have similar consideration to have the optimal solution $\left(\bar{P}_{1}, 0\right)$.

To account for the above, the optimal power control solution in (43) can be extended to

$$
\begin{aligned}
& \boldsymbol{P}_{\mathrm{opt}}^{i}=\arg \\
& \boldsymbol{P}^{i} \in\left\{\begin{array}{c}
\max _{\left(P_{1, \times}^{i, \times}, P_{2, Y}^{i, \times}\right),} \\
\left(\min \left\{\bar{P}_{1},\left.P_{1}^{i}\right|_{\left(l_{Y_{1}}, P_{2}^{i}=0\right)}\right\}, 0\right), \\
\left(0, \min \left\{\bar{P}_{2},\left.P_{2}^{i}\right|_{\left(l_{Y_{2}}, P_{1}^{i}=0\right)}\right\}\right)
\end{array}\right\} \\
& R_{\text {sum }}\left(\boldsymbol{P}^{i}\right) .
\end{aligned}
$$

Notice also that the illustration in Fig. 4 or the above analysis has assumed that the slope of $\boldsymbol{l}_{Y_{1}}<$ that of $\boldsymbol{l}_{Y_{2}}$. If the slope of $\boldsymbol{l}_{Y_{1}}>$ that of $\boldsymbol{l}_{Y_{2}}$, similar analysis can be carried out. Hence, we have the generalized result in (45) (see top of the page).

2) Scenario 2 (see Fig. 4(b)): This scenario considers that the intersection point $\boldsymbol{P}_{\mathrm{Y}}^{i, \times}$ is outside and on the right side of $\Pi$. With the assumption that the slope of $\boldsymbol{l}_{Y_{1}}<$ the slope of $\boldsymbol{l}_{Y_{2}}$, line $\boldsymbol{l}_{Y_{2}}$ solely determines the feasible region and there are two possible cases. In the first case, $l_{Y_{2}}$ cuts $P_{1}^{i}=\bar{P}_{1}$ and $P_{1}^{i}=0$ to have the intersection points $\left(\bar{P}_{1},\left.P_{2}^{i}\right|_{\left(l_{Y_{2}}, P_{1}^{i}=\bar{P}_{1}\right)}\right)$, and $\left(0,\left.P_{2}^{i}\right|_{\left(l_{Y_{2}}, P_{1}^{i}=0\right)}\right)$, respectively. Therefore, using Corollary 1 again, the points of interest for maximizing the sum-rate will be $\left(\bar{P}_{1}, 0\right)$ and $\left(0,\left.P_{2}^{i}\right|_{\left(l_{Y_{2}}, P_{1}^{i}=0\right)}\right)$. Alternatively, it may be possible that $l_{Y_{2}}$ cuts $P_{1}^{i}=\bar{P}_{1}$ and $P_{2}^{i}=\bar{P}_{2}$. In this case, the points of interests for maximizing the sum-rate become $\left(\bar{P}_{1}, 0\right)$ and $\left(0, \bar{P}_{2}\right)$. Summarizing both gives the optimal $\boldsymbol{P}_{\text {opt }}^{i}$ as

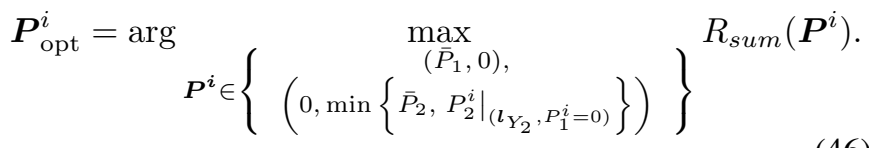

As before, after including the case that the slope of $l_{Y_{1}} \geq$ that of $l_{Y_{2}}$, the result can be generalized to (47).

3) Scenario 3 (see Fig. 4(c)): This scenario is very similar to Scenario 2 above except now that line $l_{Y_{1}}$ determines the feasible region and that the intersection $\boldsymbol{P}_{\mathrm{Y}}^{i, \times}$ is located at the top or left side of $\Pi$. One possibility is that $l_{Y_{1}}$ cuts $P_{2}^{i}=0$ and $P_{2}^{i}=\bar{P}_{2}$, which together with Corollary 1 states that the optimal power control is $\boldsymbol{P}_{\mathrm{opt}}^{i}=\left(0, \bar{P}_{2}\right)$. Another possible situation is that $l_{Y_{1}}$ cuts $P_{2}^{i}=0$ and $P_{1}^{i}=0$. In this case, the optimal power control solution should be decided between $\left(\left.P_{1}^{i}\right|_{\left(l_{Y_{1}}, P_{2}^{i}=0\right)}, 0\right)$ and $\left(0,\left.P_{2}^{i}\right|_{\left(l_{Y_{1}}, P_{1}^{i}=0\right)}\right)$. As such, combining the two cases, we have

$$
\boldsymbol{P}_{\mathrm{opt}}^{i}=\arg \boldsymbol{P}^{i} \in\left\{\begin{array}{c}
\max \\
\left(\left.P_{1}^{i}\right|_{\left(l_{Y_{1}}, P_{2}^{i}=0\right)}, 0\right), \\
\left(0, \min \left\{\bar{P}_{2},\left.P_{2}^{i}\right|_{\left(l_{Y_{1}}, P_{1}^{i}=0\right)}\right\}\right)
\end{array}\right\} R^{R_{\text {sum }}\left(\boldsymbol{P}^{i}\right) .}
$$

This result can also further be generalized to (49).

\section{With Both Data and Energy Harvesting Constraints}

Here, we consider the most general case where both the rate and energy harvesting constraints are present in maximizing the sum-rate. As worked out in the above for the date-only or energy-harvesting-only constraints cases, it becomes a matter of sorting out the feasible region (i.e., the intersection region of Lemma 2, Lemma 4 and $\Pi$ ) and within which identify the point of power allocation $\boldsymbol{P}^{i}$ that delivers the maximum sumrate. From Section III-B, we know that first the slope of $\boldsymbol{l}_{R_{1}}$ must be greater than that of $\boldsymbol{l}_{R_{2}}$ to be feasible, and that the intersection point $\boldsymbol{P}_{\mathrm{R}}^{i, \times}$ must be inside $\Pi$. Also, there will be three possible cases of how the feasible region is made out, as far as the data rate constraints are concerned. On the other hand, it is known from Section III-C that in the case of the energy harvesting constraints, there will be 8,4 and 4 possible cases for Scenario 1, 2 and 3, respectively. Actually, altogether, there are more than 48 possible shapes of the feasible region depending upon how the lines combine within $\Pi$, if both rate and energy harvesting constraints are considered. ${ }^{1}$

To begin our analysis, the following points are of interest:

\footnotetext{
${ }^{1}$ The actual total number of combinations is 76 . For details, see Appendix.
} 


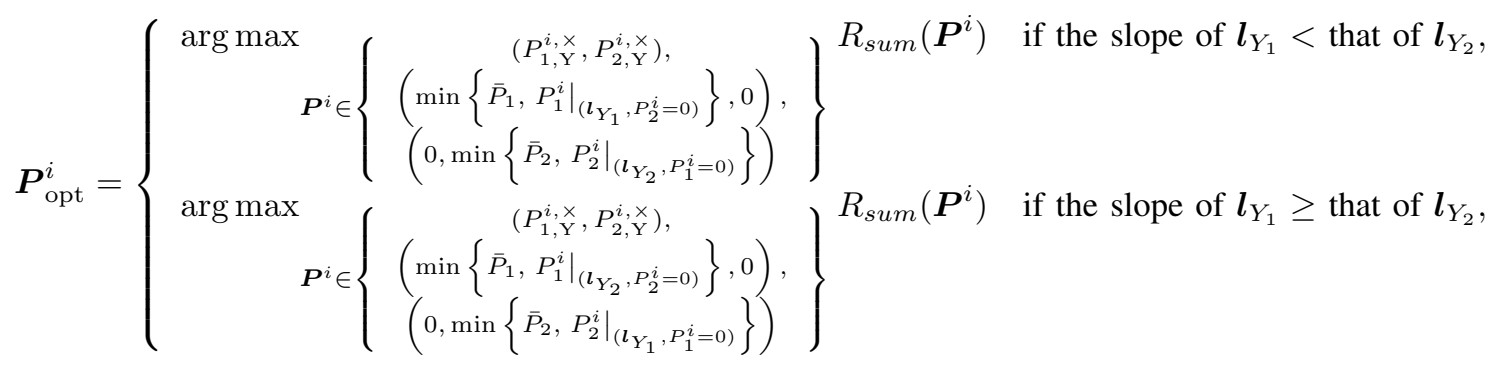

$$
\boldsymbol{P}_{\mathrm{opt}}^{i}=\left\{\begin{array}{c}
\arg \max \boldsymbol{P}^{i} \in\left\{\left(0, \min \left\{\bar{P}_{2},\left.P_{2}^{i}\right|_{\left(\boldsymbol{l}_{Y_{2}}, P_{1}^{i}=0\right)}\right\}\right)\right\} \\
\operatorname{arg\operatorname {max}} \boldsymbol{P}^{i} \in\left\{\left(0, \min \left\{\bar{P}_{2},\left.P_{2}^{i}\right|_{\left(l_{Y_{1}}, P_{1}^{i}=0\right)}\right\}\right)\right\}
\end{array}\right.
$$

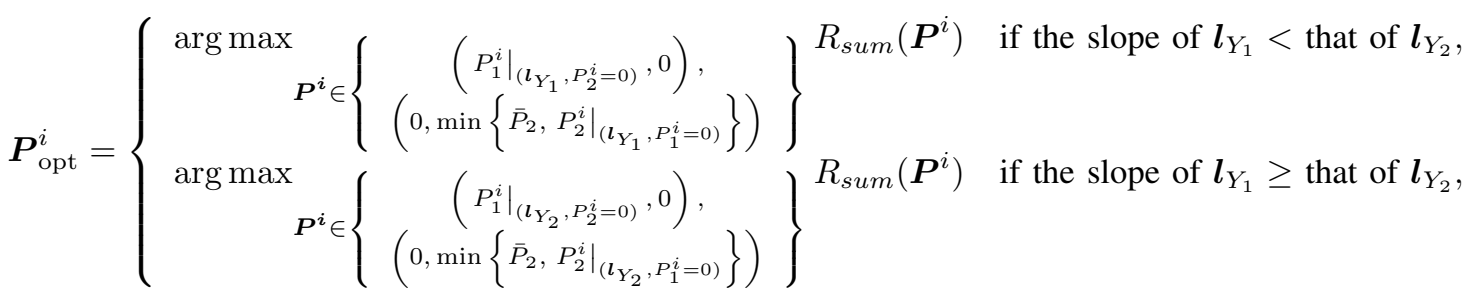

$$
\begin{aligned}
\mathrm{R}_{1, \mid} & =\left(\bar{P}_{1},\left.P_{2}\right|_{\left(\boldsymbol{l}_{R_{1}}, P_{1}^{i}=\bar{P}_{1}\right)}\right), \\
\mathrm{R}_{1,-} & =\left(\left.P_{1}\right|_{\left(\boldsymbol{l}_{R_{1}}, P_{2}^{i}=\bar{P}_{2}\right)}, \bar{P}_{2}\right), \\
\mathrm{R}_{2, \mid} & =\left(\bar{P}_{1},\left.P_{2}\right|_{\left(\boldsymbol{l}_{R_{2}}, P_{1}^{i}=\bar{P}_{1}\right)}\right), \\
\mathrm{R}_{2,-} & =\left(\left.P_{1}\right|_{\left(\boldsymbol{l}_{R_{2}}, P_{2}^{i}=\bar{P}_{2}\right)}, \bar{P}_{2}\right), \\
\mathrm{Y}_{1, \mid} & =\left(\bar{P}_{1},\left.P_{2}\right|_{\left(\boldsymbol{l}_{Y_{1}}, P_{1}^{i}=\bar{P}_{1}\right)}\right), \\
\mathrm{Y}_{1,-} & =\left(\left.P_{1}\right|_{\left(\boldsymbol{l}_{Y_{1}}, P_{2}^{i}=\bar{P}_{2}\right)}, \bar{P}_{2}\right), \\
\mathrm{Y}_{2, \mid} & =\left(\bar{P}_{1},\left.P_{2}\right|_{\left(\boldsymbol{l}_{Y_{2}}, P_{1}^{i}=\bar{P}_{1}\right)}\right), \\
\mathrm{Y}_{2,-} & =\left(\left.P_{1}\right|_{\left(\boldsymbol{l}_{Y_{2}}, P_{2}^{i}=\bar{P}_{2}\right)}, \bar{P}_{2}\right) .
\end{aligned}
$$

The above points basically mark the locations where lines $\boldsymbol{l}_{R_{1}}$, $\boldsymbol{l}_{R_{2}}, \boldsymbol{l}_{Y_{1}}$ and $\boldsymbol{l}_{Y_{2}}$ cut on the lines $P_{1}^{i}=\bar{P}_{1}$ and $P_{2}^{i}=\bar{P}_{2}$. The points may appear on the edges of the box $\Pi$ or sometimes outside $\Pi$. Furthermore, there are five points inside $\Pi$ that are important. Four of those are the intersection points among the four constraint lines, which we write them as

$$
\begin{aligned}
\mathrm{RY}_{1,1} & =\left(\left.P_{1}\right|_{\left(\boldsymbol{l}_{R_{1}}, \boldsymbol{l}_{Y_{1}}\right)},\left.P_{2}\right|_{\left(\boldsymbol{l}_{R_{1}}, \boldsymbol{l}_{Y_{1}}\right)}\right) \\
& =\left(\frac{\left(2^{\frac{1}{T-\tau}}-1\right) \frac{\mathcal{W}_{1}}{\tau}}{G_{1,1} 2^{\frac{1}{T-\tau}}}, \frac{\frac{\mathcal{W}_{1}}{\tau}-\sigma_{1}^{2} 2^{\frac{\mathcal{D}_{1}}{T-\tau}}}{G_{1,2} 2^{\frac{\mathcal{D}_{1}}{T-\tau}}}\right),
\end{aligned}
$$

$$
\begin{aligned}
\mathrm{RY}_{2,1}= & \left(\left.P_{1}\right|_{\left(\boldsymbol{l}_{R_{2}}, l_{Y_{1}}\right)},\left.P_{2}\right|_{\left(\boldsymbol{l}_{R_{2}}, l_{Y_{1}}\right)}\right) \\
= & \left(\frac{G_{2,2}\left(\frac{\mathcal{W}_{1}}{\tau}-\sigma_{1}^{2}\right)-G_{1,2}\left(2^{\frac{\mathcal{D}_{2}}{T-\tau}}-1\right) \sigma_{2}^{2}}{G_{1,1} G_{2,2}+G_{1,2} G_{2,1}\left(2^{\frac{\mathcal{D}_{2}}{T-\tau}}-1\right)},\right. \\
& \left.\frac{\left(2^{\frac{\mathcal{D}_{2}}{T-\tau}}-1\right)\left[G_{2,1}\left(\frac{\mathcal{W}_{1}}{\tau}-\sigma_{1}^{2}\right)+G_{1,1} \sigma_{2}^{2}\right]}{G_{1,1} G_{2,2}+G_{1,2} G_{2,1}\left(2^{\frac{\mathcal{D}_{2}}{T-\tau}}-1\right)}\right), \\
\mathrm{RY}_{1,2}= & \left(\left.P_{1}\right|_{\left(\boldsymbol{l}_{R_{1}}, l_{Y_{2}}\right)},\left.P_{2}\right|_{\left(\boldsymbol{l}_{R_{1}}, l_{Y_{2}}\right)}\right) \\
= & \left(\frac{\left(2^{\frac{\mathcal{D}_{1}}{T-\tau}}-1\right)\left[G_{1,2}\left(\frac{\mathcal{W}_{2}}{\tau}-\sigma_{2}^{2}\right)+G_{2,2} \sigma_{1}^{2}\right]}{G_{1,1} G_{2,2}+G_{1,2} G_{2,1}\left(2^{\frac{\mathcal{D}_{1}}{T-\tau}}-1\right)},\right. \\
& \left.\frac{G_{1,1}\left(\frac{\mathcal{W}_{2}}{\tau}-\sigma_{2}^{2}\right)-G_{2,1}\left(2^{\frac{\mathcal{D}_{1}}{T-\tau}}-1\right) \sigma_{1}^{2}}{G_{1,1} G_{2,2}+G_{1,2} G_{2,1}\left(2^{\frac{\mathcal{D}_{1}}{T-\tau}}-1\right)}\right),
\end{aligned}
$$

and

$$
\begin{aligned}
\mathrm{RY}_{2,2} & =\left(\left.P_{1}\right|_{\left(\boldsymbol{l}_{R_{2}}, \boldsymbol{l}_{Y_{2}}\right)},\left.P_{2}\right|_{\left(\boldsymbol{l}_{R_{2}}, \boldsymbol{l}_{Y_{2}}\right)}\right) \\
& =\left(\frac{\frac{\underline{\mathcal{W}}_{2}}{\tau}-\sigma_{2}^{2} 2^{\frac{\underline{\mathcal{D}}_{2}}{T-\tau}}}{G_{2,1} 2^{\frac{2}{T-\tau}}}, \frac{\left(2^{\frac{\mathcal{D}_{2}}{T-\tau}}-1\right) \frac{\mathcal{W}_{2}}{\tau}}{G_{2,2} 2^{\frac{\mathcal{D}_{2}}{T-\tau}}}\right) .
\end{aligned}
$$

Lastly, the intersection point $\boldsymbol{P}_{\mathrm{Y}}^{i, \times}$ is particularly useful, as we will use its location to analyze the optimal power allocation, $\boldsymbol{P}_{\text {opt }}^{i}$, just like what we did in Section III-B.

In the following, we will study the optimal power allocation $\boldsymbol{P}_{\text {opt }}^{i}$ by considering three possible scenarios based on where $\boldsymbol{P}_{\mathrm{Y}}^{i, \times}$ would locate. The first scenario $(i)$ investigates the case when $\boldsymbol{P}_{\mathrm{Y}}^{i, \times}$ appears inside $\Pi$, while scenario $(i i)$ considers the 
case when $\boldsymbol{P}_{\mathrm{Y}}^{i, \times}$ is outside and on the right of $\Pi$, and scenario (iii) looks into the case when $\boldsymbol{P}_{\mathrm{Y}}^{i, \times}$ is at the top or left side of $\Pi$. Each of the scenarios will be discussed next. Also, notice that in order for the problem to be feasible, the intersection of the regions specified in Lemma 2 and Lemma 4 must not be empty, which we will assume when we proceed. In addition, for convenience, we will assume that the slope of $l_{Y_{1}}$ is less than that of $l_{Y_{2}}$, but our analysis can be easily extended by swapping the indices corresponding to lines $\boldsymbol{l}_{Y_{1}}$ and $\boldsymbol{l}_{Y_{2}}$.

1) Scenario (i): Even within the case where $\boldsymbol{P}_{\mathrm{Y}}^{i, \times}$ is inside $\Pi$, there are many possibilities which would affect the finding of $\boldsymbol{P}_{\text {opt }}^{i}$. To start with, we study the mixing of Fig. 4(a) and the various possible shapes of the feasible region of the rate constraints. In fact, Fig. 4(a) represents as many as 8 possible cases $\left(\boldsymbol{l}_{Y_{2}}\right.$ cuts either $P_{1}^{i}=0$ or $P_{2}^{i}=\bar{P}_{2} ; \boldsymbol{l}_{Y_{1}}$ cuts $P_{2}^{i}=0$ or $P_{1}^{i}=\bar{P}_{1}$; and $\boldsymbol{l}_{Y_{1}}$ and $\boldsymbol{l}_{Y_{2}}$ swap). Let us first restrict ourselves to the case $(a)$ if $\boldsymbol{l}_{Y_{2}}$ cuts $P_{2}^{i}=\bar{P}_{2}$ and $\boldsymbol{l}_{Y_{1}}$ cuts $P_{1}^{i}=\bar{P}_{1}$, so the feasible region due to the energy harvesting constraints makes out a hexagon, with 4 sides there to be cut by $\boldsymbol{l}_{R_{1}}$ and $l_{R_{2}}$. As shown in the appendix, there are 10 ways of lines $\boldsymbol{l}_{R_{1}}$ and $l_{R_{2}}$ landing on the four edges, which we will list below. Before we do so, note that in all the cases, the optimal power allocation pairs $\boldsymbol{P}_{\mathrm{opt}}^{i}$ can be commonly obtained by

$$
\boldsymbol{P}_{\mathrm{opt}}^{i}=\arg \max _{\boldsymbol{P}^{i} \in \Pi^{*}} R_{\text {sum }}\left(\boldsymbol{P}^{i}\right),
$$

where $\Pi^{*}$ denotes the set of points with potential to be the optimum. Thus, henceforth, our focus is on finding the set $\Pi^{*}$.

Now, we list the 10 cases for scenario $(i)$ as follows:

- $(1,1)$, i.e., $\left.P_{2}\right|_{\mathrm{RY}_{1,2}}>\bar{P}_{2}$ and $\left.P_{2}\right|_{\mathrm{RY}_{2,2}}>\bar{P}_{2} .^{2}$ In this case, the rate constraints dominate (i.e., satisfying the rate constraints implies satisfaction of the energy harvesting constraints) and the optimal $\boldsymbol{P}_{\mathrm{opt}}^{i}$ is given by (39).

- $(1,2)$, i.e., $\left.P_{2}\right|_{\mathrm{RY}_{1,2}}>\bar{P}_{2}$ and $\left.P_{2}\right|_{P_{Y}^{i, \times}}<\left.P_{2}\right|_{\mathrm{RY}_{2,2}}<\bar{P}_{2}$. As a result, the set $\Pi^{*}$ can be found as

$$
\Pi^{*}=\left\{\mathrm{R}_{1,-}, \mathrm{Y}_{2,-}, \mathrm{RY}_{2,2}\right\} .
$$

- $(1,3):\left.P_{2}\right|_{\mathrm{RY}_{1,2}}>\bar{P}_{2}$ and $\left.P_{2}\right|_{\mathrm{Y}_{1,1}}<\left.P_{2}\right|_{\mathrm{RY}_{2,1}}<\left.P_{2}\right|_{P_{\mathrm{Y}}^{i, \times}}$. Therefore, $\Pi^{*}$ is determined as

$$
\Pi^{*}=\left\{\mathrm{R}_{1,-}, \mathrm{Y}_{2,-}, \boldsymbol{P}_{\mathrm{Y}}^{i, \times}, \mathrm{RY}_{2,1}\right\} .
$$

- $(1,4):\left.P_{2}\right|_{\mathrm{RY}_{1,2}}>\bar{P}_{2}$ and $\left.P_{2}\right|_{\mathrm{R}_{2, \mid}}<\left.P_{2}\right|_{\mathrm{Y}_{1, \mid}}$, which gives

$$
\Pi^{*}=\left\{\mathrm{R}_{1,-}, \mathrm{Y}_{2,-}, \boldsymbol{P}_{\mathrm{Y}}^{i, \times}, \mathrm{Y}_{1, \mid}, \mathrm{R}_{2, \mid}\right\} .
$$

- $(2,2)$ : That is, $\left.P_{2}\right|_{P_{\mathrm{Y}}^{i, \times}}<\left.P_{2}\right|_{\mathrm{RY}_{1,2}}<\bar{P}_{2}$ and $\left.P_{2}\right|_{P_{\mathrm{Y}}^{i, \times}}<$ $\left.P_{2}\right|_{\mathrm{RY}_{2,2}}<\bar{P}_{2}$. In this case, we have

$$
\Pi^{*}=\left\{\mathrm{RY}_{1,2}, \mathrm{RY}_{2,2}\right\} \text {. }
$$

- $(2,3)$ : That is, $\left.P_{2}\right|_{P_{\mathrm{Y}}^{i, \times}}<\left.P_{2}\right|_{\mathrm{RY}_{1,2}}<\bar{P}_{2}$ and $\left.P_{2}\right|_{\mathrm{Y}_{1, \mid}}<$ $\left.P_{2}\right|_{\mathrm{RY}_{2,1}}<\left.P_{2}\right|_{P_{\mathrm{Y}}^{i, \times}}$. Then $\Pi^{*}$ is given by

$$
\Pi^{*}=\left\{\mathrm{RY}_{1,2}, \boldsymbol{P}_{\mathrm{Y}}^{i, \times}, \mathrm{RY}_{2,1}\right\} \text {. }
$$

\footnotetext{
${ }^{2}$ Here, the notation $P_{m} \mid \mathrm{X}$ indicates the $P_{m}$ coordinate for point $\mathrm{X}$.
}

- $(2,4)$ : That is, $\left.P_{2}\right|_{P_{\mathrm{Y}}^{i, \times}}<\left.P_{2}\right|_{\mathrm{RY}_{1,2}}<\bar{P}_{2}$ and $\left.P_{2}\right|_{\mathrm{R}_{2, \mid}}<$ $\left.P_{2}\right|_{Y_{1,1}}$. Consequently, we have $\Pi^{*}$ given by

$$
\Pi^{*}=\left\{\mathrm{RY}_{1,2}, \boldsymbol{P}_{\mathrm{Y}}^{i, \times}, \mathrm{Y}_{1, \mid}, \mathrm{R}_{2, \mid}\right\} .
$$

- $(3,3)$ : That is, $\left.P_{2}\right|_{\mathrm{Y}_{1,1}}<\left.P_{2}\right|_{\mathrm{RY}_{1,1}},\left.P_{2}\right|_{\mathrm{RY}_{2,1}}<\left.P_{2}\right|_{P_{\mathrm{Y}}^{i, \times}}$. As such, the set $\Pi^{*}$ is found as

$$
\Pi^{*}=\left\{\mathrm{RY}_{1,1}, \mathrm{RY}_{2,1}\right\} .
$$

- $(3,4)$ : That is, $\left.P_{2}\right|_{\mathrm{Y}_{1,1}}<\left.P_{2}\right|_{\mathrm{RY}_{1,1}}<\left.P_{2}\right|_{P_{\mathrm{Y}}^{i, \times}}$ as well as $\left.P_{2}\right|_{\mathrm{R}_{2,1}}<\left.P_{2}\right|_{\mathrm{Y}_{1, \mid}}$. As a result, we get

$$
\Pi^{*}=\left\{\mathrm{RY}_{1,1}, \mathrm{Y}_{1, \mid}, \mathrm{R}_{2, \mid}\right\} .
$$

- $(4,4)$ : That is, $\left.P_{2}\right|_{\mathrm{R}_{1,1}},\left.P_{2}\right|_{\mathrm{R}_{2, \mid}}<\left.P_{2}\right|_{\mathrm{Y}_{1,1}}$. Hence,

$$
\Pi^{*}=\left\{\mathrm{R}_{1, \mid}, \mathrm{R}_{2, \mid}\right\} .
$$

Note that $\boldsymbol{l}_{Y_{2}}$ may cut $P_{1}^{i}=0$ instead of $P_{2}^{i}=\bar{P}_{2}$, while $\boldsymbol{l}_{Y_{1}}$ still cuts $P_{1}^{i}=\bar{P}_{1}$. We refer to this case as $(b)$. In this case, we will have a pentagon feasible region made out by the energy harvesting constraints and have the following 6 situations:

- $(1,1)$ : This case is same as $(2,2)$ in case $(a)$ above.

- $(1,2)$ : This case is same as $(2,3)$ in case $(a)$ above.

- $(1,3)$ : This case is same as $(2,4)$ in case $(a)$ above.

- $(2,2)$ : This case is same as $(3,3)$ in case $(a)$ above.

- $(2,3)$ : This case is same as $(3,4)$ in case $(a)$ above.

- $(3,3)$ : This case is same as $(4,4)$ in case $(a)$ above.

We can also have the case $(c)$ that $\boldsymbol{l}_{Y_{2}}$ may cut $P_{2}^{i}=\bar{P}_{2}$, but $l_{Y_{1}}$ cuts $P_{2}^{i}=0$ instead. In this case, we will have a pentagon feasible region and have the following 6 situations:

- $(1,1)$ : This case is same as $(1,1)$ in case $(a)$ above.

- $(1,2)$ : This case is same as $(1,2)$ in case $(a)$ above.

- $(1,3)$ : This case is same as $(1,3)$ in case $(a)$ above.

- $(2,2)$ : This case is same as $(2,2)$ in case $(a)$ above.

- $(2,3)$ : This case is same as $(2,3)$ in case $(a)$ above.

- $(3,3)$ : This case is same as $(3,3)$ in case $(a)$ above.

The last case $(d)$ is that $\boldsymbol{l}_{Y_{2}}$ may cut $P_{1}^{i}=0$, and $\boldsymbol{l}_{Y_{1}}$ cuts $P_{2}^{i}=0$. In this case, we will only have 3 situations:

- $(1,1)$ : This case is same as $(2,2)$ in case $(a)$ above.

- $(1,2)$ : This case is same as $(2,3)$ in case $(a)$ above.

- $(2,2)$ : This case is same as $(3,3)$ in case $(a)$ above.

2) Scenario (ii): Here, we address the case when $\boldsymbol{P}_{\mathrm{Y}}^{i, \times}$ is outside and on the right of $\Pi$. There are two possible cases in scenario $(i i)$. We first look at case $(a)$ if $\boldsymbol{l}_{Y_{2}}$ cuts $P_{2}^{i}=\bar{P}_{2}$ and $P_{1}^{i}=\bar{P}_{1}$. In this case, we have a pentagon feasible region and have the following 6 situations:

- $(1,1)$ : This case is same as $(1,1)$ in scenario $(i)(a)$.

- $(1,2)$ : The condition needs to be changed to $\left.P_{2}\right|_{\mathrm{RY}_{1,2}}>$ $\bar{P}_{2}$ and $\left.P_{2}\right|_{\mathrm{Y}_{2,1}}<\left.P_{2}\right|_{\mathrm{RY}_{2,2}}<\bar{P}_{2}$, although the optimal set $\Pi^{*}$ is same as $(1,2)$ in scenario $(i)(a)$, or $(64)$.

- $(1,3)$ : That is, $\left.P_{2}\right|_{\mathrm{RY}_{1,2}}>\bar{P}_{2}$ and $\left.P_{2}\right|_{\mathrm{R}_{2,1}}<\left.P_{2}\right|_{\mathrm{Y}_{2,1}}$. As a result, $\Pi^{*}$ is given by

$$
\Pi^{*}=\left\{\mathrm{R}_{1,-}, \mathrm{Y}_{2,-}, \mathrm{Y}_{2, \mid}, \mathrm{R}_{2, \mid}\right\} .
$$

- $(2,2)$ : The optimal set $\Pi^{*}$ is given by the result of $(2,2)$ in scenario $(i)(a)$, i.e., (67), but the condition has now been revised to $\left.P_{2}\right|_{\mathrm{Y}_{2,1}}<\left.P_{2}\right|_{\mathrm{RY}_{1,2}},\left.P_{2}\right|_{\mathrm{RY}_{2,2}}<\bar{P}_{2}$. 
- $(2,3)$ : That is, $P_{2}\left|\mathrm{Y}_{2,1}<P_{2}\right|_{\mathrm{RY}_{1,2}}<\bar{P}_{2}$ and $\left.P_{2}\right|_{\mathrm{R}_{2,1}}<$ $\left.P_{2}\right|_{\mathrm{Y}_{2,1}}$. Then $\Pi^{*}$ is given by

$$
\Pi^{*}=\left\{\mathrm{RY}_{1,2}, \mathrm{Y}_{2, \mid}, \mathrm{R}_{2, \mid}\right\} .
$$

- $(3,3)$ : The condition is $\left.P_{2}\right|_{\mathrm{R}_{1,1}},\left.P_{2}\right|_{\mathrm{R}_{2,1}}<\left.P_{2}\right|_{\mathrm{Y}_{2, \mid}}$ but the set $\Pi^{*}$ is same as $(4,4)$ in scenario $(i)(a)$ or $(72)$.

Now, we move on to another case $(b)$ in which $\boldsymbol{l}_{Y_{2}}$ still cuts $P_{1}^{i}=\bar{P}_{1}$ but opts to cut $P_{1}^{i}=0$ instead of $P_{2}^{i}=\bar{P}_{2}$. In that case, we will only have 3 possibilities:

- $(1,1)$ : This case is same as $(2,2)$ in scenario $(i i)(a)$.

- $(1,2)$ : This case is same as $(2,3)$ in scenario $(i i)(a)$.

- $(2,2)$ : This case is same as $(3,3)$ in scenario $(i i)(a)$.

3) Scenario (iii): This scenario looks into the case when $\boldsymbol{P}_{\mathrm{Y}}^{i, \times}$ is at the top or left side of $\Pi$, which corresponds to the fact that $(a) l_{Y_{1}}$ cuts $P_{2}^{i}=\bar{P}_{2}$ and $P_{2}^{i}=0$, or $(b) l_{Y_{1}}$ cuts $P_{1}^{i}=0$ and $P_{2}^{i}=0$. For $(a)$, we have 3 situations:

- $(1,1)$ : Same as in scenario $(i)(a)$, the optimal $\boldsymbol{P}_{\mathrm{opt}}^{i}$ can be found by (39), and $\Pi^{*}$ is given by $\left\{R_{1,-}, R_{2,-}\right\}$ but the condition becomes $\left.P_{2}\right|_{\mathrm{RY}_{1,1}},\left.P_{2}\right|_{\mathrm{RY}_{2,1}}>\bar{P}_{2}$.

- $(1,2)$ : That is, $\left.P_{2}\right|_{\mathrm{RY}_{1,1}}>\bar{P}_{2}$ and $\left.P_{2}\right|_{\mathrm{RY}_{2,1}}<\bar{P}_{2}$. The set $\Pi^{*}$ therefore can be obtained as

$$
\Pi^{*}=\left\{\mathrm{R}_{1,-}, \mathrm{Y}_{1,-}, \mathrm{RY}_{2,1}\right\} .
$$

- $(2,2)$ : That is, $\left.P_{2}\right|_{\mathrm{RY}_{1,1}},\left.P_{2}\right|_{\mathrm{RY}_{2,1}}<\bar{P}_{2}$. The optimal set $\Pi^{*}$ is given by $(70)$ in $(3,3)$ of scenario $(i)(a)$.

On the other hand, for scenario $(i i i)(b)$, we will only have one possibility with a triangular feasible region from the energy harvesting constraints. In that case, the result is same as $(2,2)$ in scenario $(i i i)(a)$ above for finding the optimal set $\Pi^{*}$.

\section{Simulation Results}

In this section, numerical examples are presented to illustrate how the optimal power allocation and its corresponding sum-rate vary w.r.t. the constraints, and how the feasible region changes its shape to validate our analysis. For convenience, we set $\bar{P}_{1}=\bar{P}_{2}=2$ and $\sigma_{1}^{2}=\sigma_{2}^{2}=0.01$, and as a reference, also define the signal-to-noise ratio (SNR) as

$$
\mathrm{SNR}=10 \log _{10} \frac{\bar{P}_{1}}{\sigma_{1}^{2}}=10 \log _{10} \frac{2}{0.01}=23 \mathrm{~dB} .
$$

Fig. 8 assumed the following channel gain matrix

$$
\mathbf{G}=\left[\begin{array}{ll}
0.7323 & 0.0451 \\
0.0366 & 0.2600
\end{array}\right]
$$

and considered the constraints $\frac{\underline{\mathcal{D}}_{1}}{T-\tau}=2.5, \frac{\underline{\mathcal{D}}_{2}}{T-\tau}=2.8, \frac{\mathcal{W}_{1}}{\tau}=$ 0.25 , and $\frac{\mathcal{W}_{2}}{\tau}=0.4$. The optimal points with and without the constraints are marked in the figure. Fig. 9 provided similar results but with a different channel gain matrix

$$
\mathbf{G}=\left[\begin{array}{ll}
0.1942 & 0.0213 \\
0.0229 & 0.8234
\end{array}\right]
$$

and $\frac{\underline{\mathcal{D}}_{1}}{T-\tau}=2.2, \frac{\mathcal{D}_{2}}{T-\tau}=2.8, \frac{\mathcal{W}_{1}}{\tau}=0.2, \frac{\mathcal{W}_{2}}{\tau}=0.4$. As we can see, without rate and energy-harvesting constraints, the optimal point for the example in Fig. 8 appears as $\left(\bar{P}_{1}, 0\right)$, while for Fig. 9, it appears as $\left(\bar{P}_{1}, \bar{P}_{2}\right)$, which aligns with our analysis. Table III provides the sum-rates for the two examples.

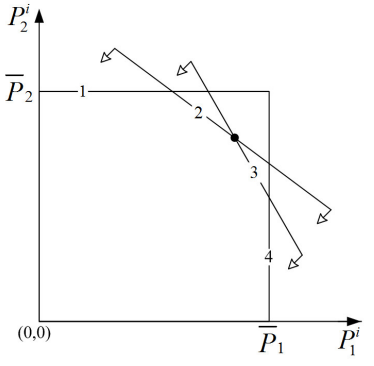

(a) Scenario $(i)(a)$

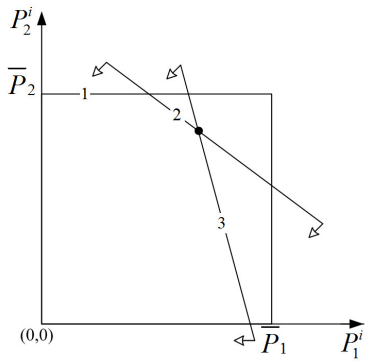

(c) Scenario $(i)(c)$

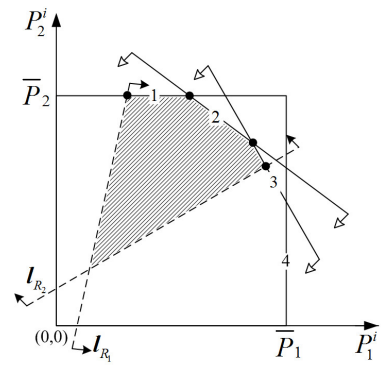

(e) $(1,3)$ of scenario $(i)(a)$

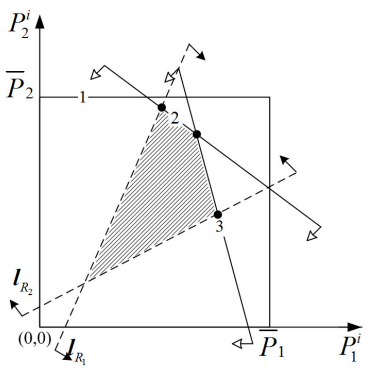

(g) $(2,3)$ of scenario $(i)(c)$

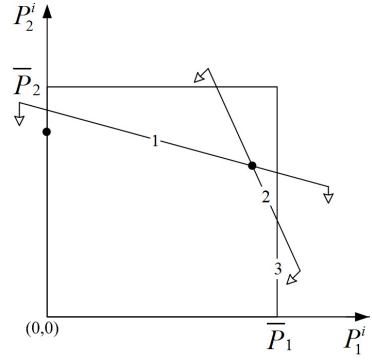

(b) Scenario $(i)(b)$

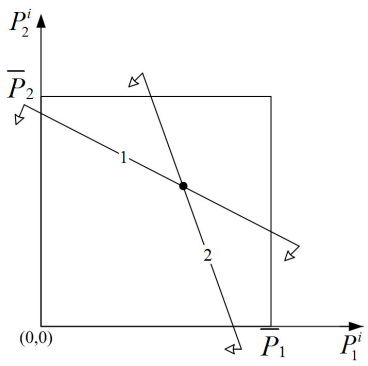

(d) Scenario $(i)(d)$

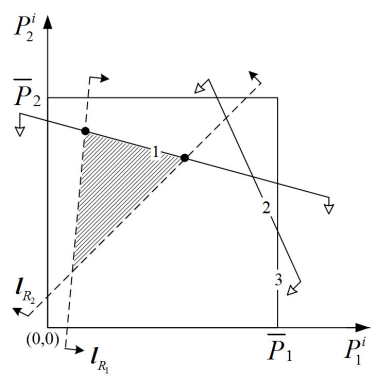

(f) $(1,1)$ of scenario $(i)(b)$

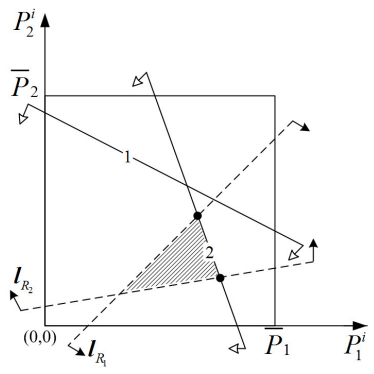

(h) $(2,2)$ of scenario $(i)(d)$
Fig. 5. Illustration of the possible combinations of lines $\boldsymbol{l}_{R_{1}}, \boldsymbol{l}_{R_{2}}, \boldsymbol{l}_{l_{1}}$ and $\boldsymbol{l}_{l_{2}}$ for scenario $(i)$ where $\boldsymbol{P}_{\mathrm{Y}}^{i, \times}$ is inside $\Pi$. In (a)-(d), it shows 4 possible ways $\boldsymbol{l}_{Y_{1}}$ and $\boldsymbol{l}_{Y_{2}}$ may cut $\Pi$ to form the region due to the energy harvesting constraints with numbered edges, while (e)-(h) provide examples for each of the cases how $l_{R_{1}}$ and $l_{R_{2}}$ may cut the edges to form the feasible region.

TABLE III

SUM-RATES FOR THE TIME-SWITCHING SWIPT SYSTEM.

\begin{tabular}{r||c|c|c|c}
\hline $\begin{array}{r}\text { Sum-rate } \\
\text { (bits/s/Hz) }\end{array}$ & $\begin{array}{r}\text { No } \\
\text { constraint }\end{array}$ & $\begin{array}{c}\text { Only WIT } \\
\text { constraints }\end{array}$ & $\begin{array}{c}\text { Only WET } \\
\text { constraints }\end{array}$ & $\begin{array}{r}\text { All } \\
\text { constraints }\end{array}$ \\
\hline Fig. 8 & 7.2043 & $\begin{array}{r}6.8210 \\
(94.7 \%)\end{array}$ & $\begin{array}{r}7.0454 \\
(97.8 \%)\end{array}$ & $\begin{array}{r}6.2408 \\
(86.6 \%)\end{array}$ \\
\hline Fig. 9 & 7.9973 & $\begin{array}{r}7.9973 \\
(100 \%)\end{array}$ & $\begin{array}{r}7.7866 \\
(97.3 \%)\end{array}$ & $\begin{array}{r}7.7866 \\
(97.3 \%)\end{array}$ \\
\hline
\end{tabular}



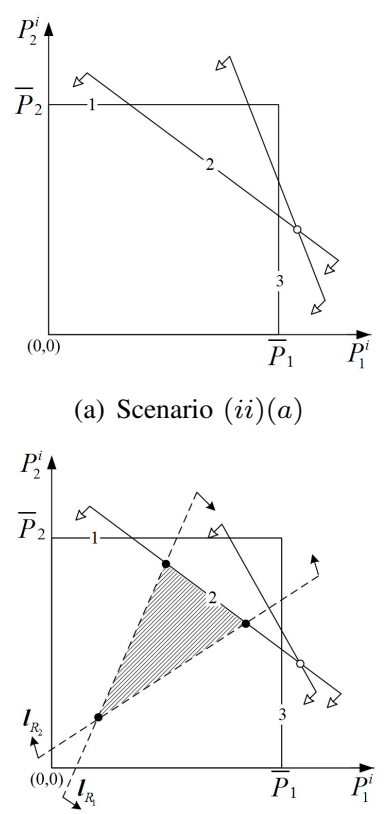

(c) $(2,2)$ of scenario $(i i)(a)$ (a) Scenario $(i i)(a)$

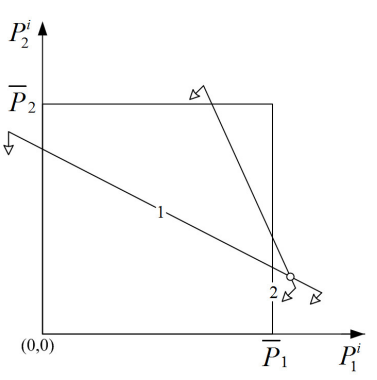

(b) Scenario $(i i)(b)$

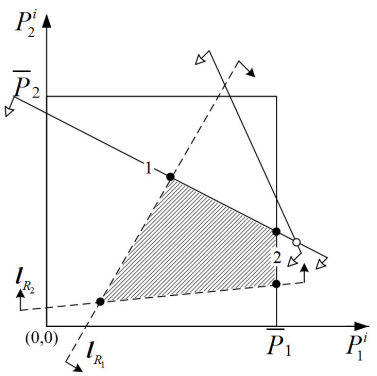

(d) $(1,2)$ of scenario $(i i)(b)$
Fig. 6. Illustration of the possible combinations of lines $\boldsymbol{l}_{R_{1}}, \boldsymbol{l}_{R_{2}}, \boldsymbol{l}_{l_{1}}$ and $\boldsymbol{l}_{l_{2}}$ for scenario (ii) where $\boldsymbol{P}_{Y}^{i, \times}$ is outside and on the right of $\Pi$. In (a) and (b), it shows 2 possible ways $\boldsymbol{l}_{Y_{1}}$ and $\boldsymbol{l}_{Y_{2}}$ may cut $\Pi$ while in (c) and (d), it shows examples of how $\boldsymbol{l}_{R_{1}}, \boldsymbol{l}_{R_{2}}$ cut the edges to form the feasible region.

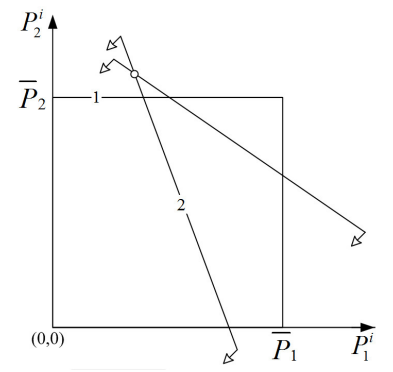

(a) Scenario $($ iii $)(a)$

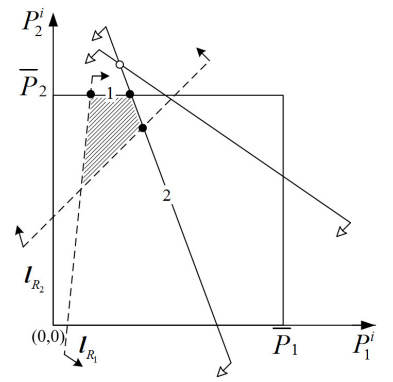

(c) $(1,2)$ of scenario $(i i i)(a)$

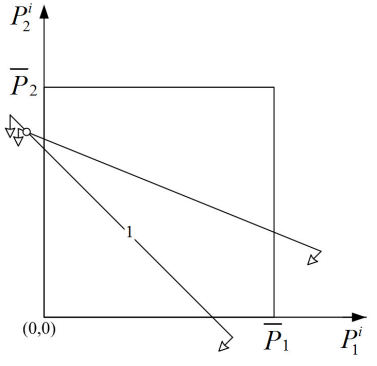

(b) Scenario $(i i i)(b)$

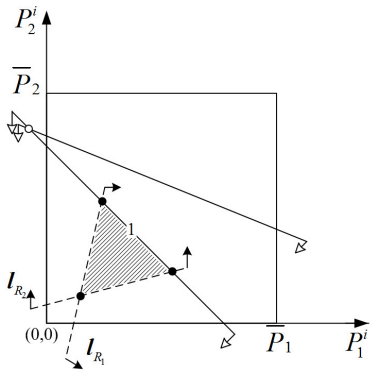

(d) $(1,1)$ of scenario $(i i i)(b)$
Fig. 7. Illustration of the possible combinations of lines $\boldsymbol{l}_{R_{1}}, \boldsymbol{l}_{R_{2}}, \boldsymbol{l}_{l_{1}}$ and $\boldsymbol{l}_{l_{2}}$ for scenario (iii) where $\boldsymbol{P}_{\mathrm{Y}}^{i, \times}$ is at the top or left side of $\Pi$. In (a) and (b), it shows 2 possible ways $\boldsymbol{l}_{Y_{1}}$ and $\boldsymbol{l}_{Y_{2}}$ may cut $\Pi$ while in (c) and (d), it shows examples of how $\boldsymbol{l}_{R_{1}}, \boldsymbol{l}_{R_{2}}$ cut the edges to form the feasible region.

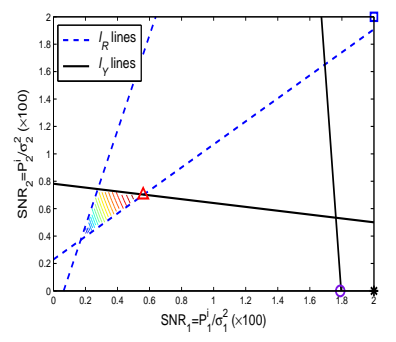

(a) The resulting feasible region

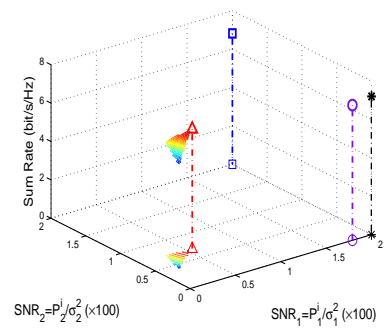

(b) Comparison of sum-rates

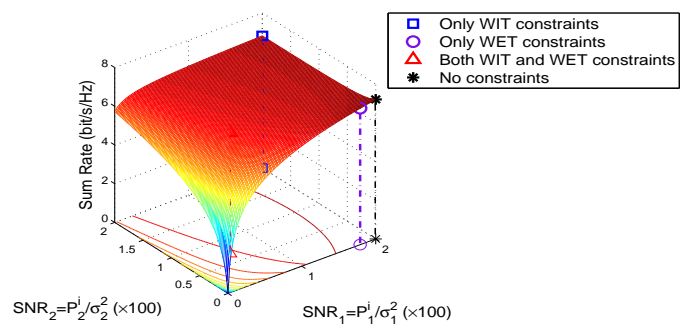

(c) Sum-rate versus SNR

Fig. 8. Results for the time-switching SWIPT system for a given $\mathbf{G}$.

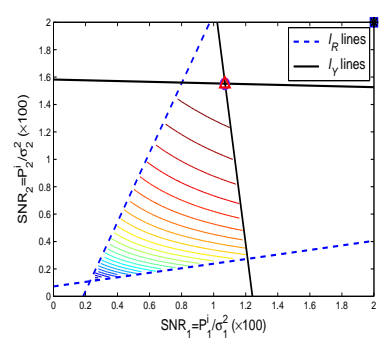

(a) The resulting feasible region

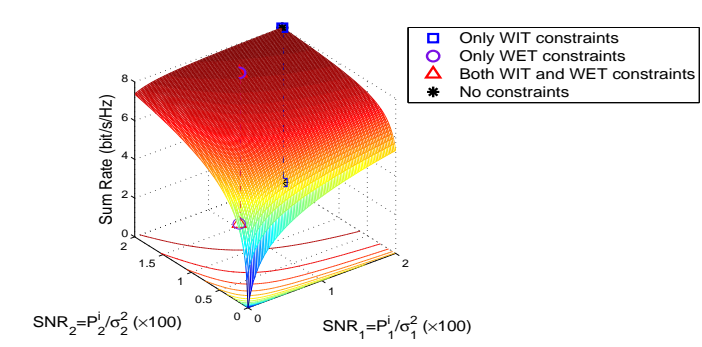

(c) Sum-rate versus SNR

Fig. 9. Results for the time-switching SWIPT system for another $\mathbf{G}$.

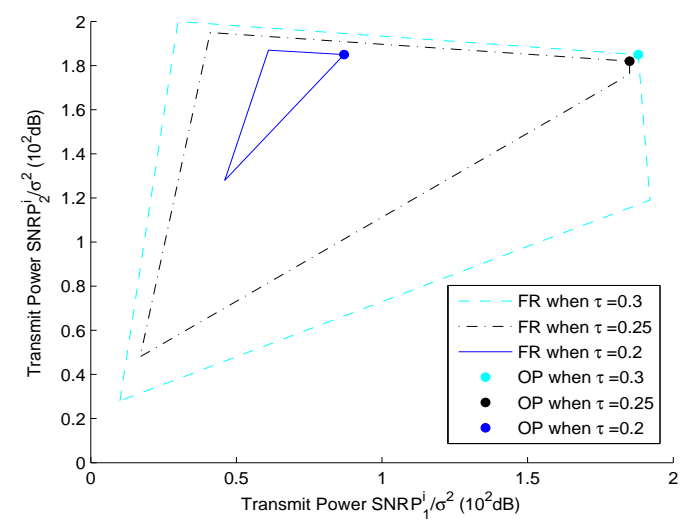

Fig. 10. Feasible region versus $\tau$.

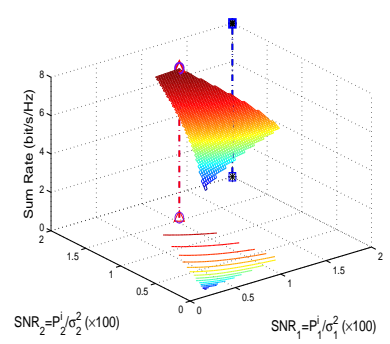

(b) Comparison of sum-rates 


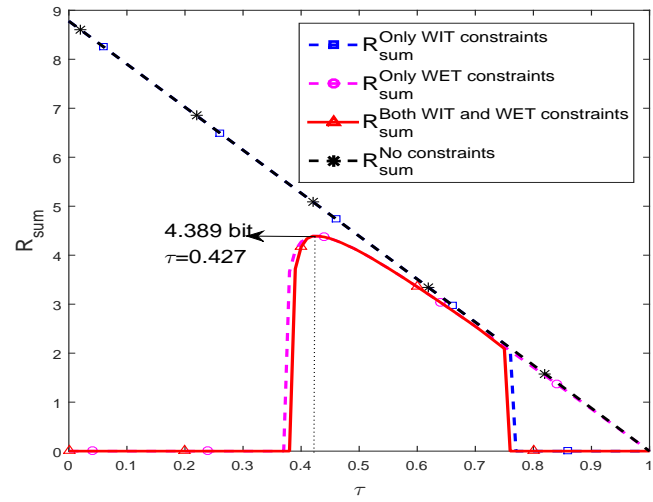

Fig. 11. The sum-rates versus the time-switching factor $\tau$.

We now proceed to illustrate how the feasible region varies w.r.t. the time allocation parameter $\tau$. The results are shown in Fig. 10 in which we have assumed that

$$
\mathbf{G}=\left[\begin{array}{ll}
0.3252 & 0.0172 \\
0.0221 & 0.2379
\end{array}\right],
$$

and $\underline{\mathcal{D}}_{1}=0.5, \underline{\mathcal{D}}_{2}=0.8, \underline{\mathcal{W}}_{1}=0.015$, and $\underline{\mathcal{W}}_{2}=0.014$, assuming $T=1$. Results for $\tau=0.3,0.25,0.2$ are shown. It can be observed that the feasible region as well as the sumrate optimal point change with $\tau$. An optimization of $\tau$ can only be achieved using numerical methods.

Even though the joint optimization of the power allocation strategy and $\tau$ does not admit any closed-form solution, our derived closed-form power allocation solution greatly facilitates the optimization using a simple one-dimensional search. To help illustrate the joint optimization with $\tau$, Fig. 11 shows the sum-rate against the various value of $\tau$ using an example assuming the channel gain matrix

$$
\mathbf{G}=\left[\begin{array}{cc}
0.9404 & 0.0273 \\
0.0410 & 0.6250
\end{array}\right]
$$

and with the constraints being $\underline{\mathcal{D}}_{1}=1, \underline{\mathcal{D}}_{2}=1, \underline{\mathcal{W}}_{1}=0.5$, and $\underline{\mathcal{W}}_{2}=0.5$. The results demonstrate how the sum-rates may vary with and without the rate and energy harvesting constraints. In this particular example, with both rate and energy harvesting constraints, it can be observed that the sumrate attains its maximum $R_{\text {sum }}=4.389$ when $\tau=0.427$.

While this paper focuses mainly on the interference channels with time-switching WIT and WPT, one may presume that for the two-user channel, the sum-rates can be furthermore maximized by alternating WIT and WPT between the two users. That is to say, when user 1 adopts WIT, user 2 operates in the WPT mode, and vice versa. Nonetheless, it is not clear that the alternating approach would definitely perform better in the sum-rate maximization problem with rate and energy harvesting constraints than our considered approach.

In order to gain more insights, we consider the alternating approach and note that for the first time instance with duration $T-\tau$, it would have

$$
\left\{\begin{array}{l}
\mathcal{D}_{1}=(T-\tau) \log _{2}\left(1+\frac{P_{1}^{i} G_{1,1}}{\sigma_{n}^{2}+P_{2}^{e} G_{1,2}}\right), \\
\mathcal{W}_{2}=(T-\tau)\left(P_{2}^{e} G_{2,2}+\sigma_{2}^{2}+P_{1}^{i} G_{2,1}\right) .
\end{array}\right.
$$

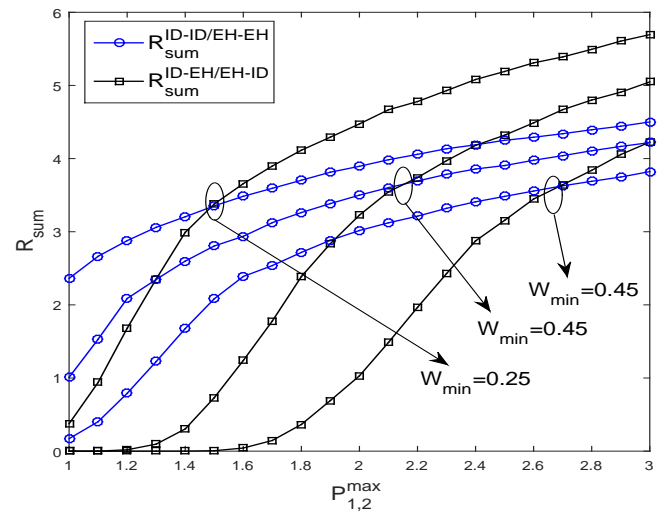

Fig. 12. The sum rates with both rate and energy harvesting constraints against the power budget $\bar{P}_{1,2}$, with $\tau=0.5$.

Then for the second instance with duration $\tau$, it has

$$
\left\{\begin{array}{l}
\mathcal{W}_{1}=\tau\left(P_{1}^{e} G_{1,1}+\sigma_{1}^{2}+P_{2}^{i} G_{1,2}\right), \\
\mathcal{D}_{2}=\tau \log _{2}\left(1+\frac{P_{2}^{i} G_{2,2}}{\sigma_{n}^{2}+P_{1}^{e} G_{2,1}}\right) .
\end{array}\right.
$$

In Fig. 12, we illustrate the average sum-rates over 1000 independent channel realizations with $\underline{\mathcal{D}}_{1,2}=1.2$, and compare them with our optimal solution for the interference system. As can be observed, for smaller peak power, the interference system indeed has higher sum-rates than the alternating model, although as the peak power increases, the sum-rates for the alternating model begin to benefit and result in higher rates. In addition, the crossover points will appear later, or at larger peak power, as the energy harvesting constraints become more stringent. The numerical results have now confirmed that the alternating model is actually not necessarily more beneficial than the interference model in the paper.

\section{CONClusion}

This paper studied the optimization problem for the power allocation for a 2-user interference channel for time-switching SWIPT. With both the rate and energy harvesting constraints, the sum-rate has been maximized by investigating the geometric properties of the constraints assuming perfect CSI.

\section{APPENDIX}

In this appendix, we will list the possible cases for making out the feasible region for the power allocation when both the rate and energy harvesting constraints are considered. We will also describe how we refer to particular cases by numbering the edges of $\boldsymbol{l}_{Y_{1}}$ and $\boldsymbol{l}_{Y_{2}}$. Note that throughout we will assume that the slope of $\boldsymbol{l}_{Y_{2}}$ is greater than that of $\boldsymbol{l}_{Y_{1}}$ for conciseness. All the results will apply naturally by swapping the indices corresponding to lines $\boldsymbol{l}_{Y_{1}}$ and $\boldsymbol{l}_{Y_{2}}$, if it is not.

Figs. 5(a)-(d) illustrate all 4 possible cases of the feasible region made out by the energy harvesting constraints when $\boldsymbol{P}_{\mathrm{Y}}^{i, \times}$ is inside $\Pi^{*}$, which we refer to them, respectively, as scenario $(i)(a)$ to $(i)(d)$, while Figs. 5(e)-(h) provide, for each of the cases, an example how lines $\boldsymbol{l}_{R_{1}}$ and $\boldsymbol{l}_{R_{2}}$ may cut on the edges of the region to form the resultant feasible region. To distinguish the various cases, we number the edges of the 
region made out by lines $\boldsymbol{l}_{Y_{1}}$ and $\boldsymbol{l}_{Y_{2}}$. For example, in Fig. 5(d), $\boldsymbol{l}_{R_{1}}$ cuts edge 1 and $\boldsymbol{l}_{R_{2}}$ cuts edge 3 . Hence, it is referred to as the $(1,3)$ case in scenario $(i)(a)$. Other examples and cases can be understood in the same way. As a result, we can also see that Figs. 5(a)-(d) represent 25 cases in total.

Moreover, Figs. 6 and 7 provide the illustrations for scenario (ii) when $\boldsymbol{P}_{\mathrm{Y}}^{i, \times}$ is outside and on the right of $\Pi^{*}$ and (iii) if $\boldsymbol{P}_{\mathrm{Y}}^{i, \times}$ is at the top or left side of $\Pi^{*}$. We can see that scenario (ii) create a total of 9 cases while scenario (iii) could make out 4 possible feasible regions. Scenarios $($ i $)-($ iii $)$ altogether thus give a total of $38 \times 2=76$ possible cases, after we take into account of the fact that lines $\boldsymbol{l}_{Y_{1}}$ and $\boldsymbol{l}_{Y_{2}}$ can swap.

\section{ACKNOWLEDGEMENT}

The authors wish to thank: 1, Shenzhen Key Laboratory of Artificial Microstructure Design(No. CXB201109210099A); Shenzhen Kuang-Chi Institute of Advanced Technology; 2, Supported by Guangdong Innovative Research Team Program(No. 2009010005); Shenzhen Kuang-Chi Institute of Advanced Technology; 3, Guangdong Key Laboratory of MetaRF Microwave Radio Frequency(No. 2011A060901010); Shenzhen Kuang-Chi Institute of Advanced Technology.

\section{REFERENCES}

[1] C. Han, T. Harrold, S. Armour, I. Krikidis, S. Videv, P. M. Grant, H. Haas, J. S. Thompson, I. Ku, C.-X. Wang, T. A. Le, M. R. Nakhai, J. Zhang, and L. Hanzo, "Green radio: Radio techniques to enable energyefficient wireless networks," IEEE Commun. Mag., vol. 49, no. 6, pp. 46-54, Jun. 2011.

[2] L. R. Varshney, "Transporting information and energy simultaneously," in Proc. IEEE Int. Symp. Inf. Theory, Jul. 2008, pp. 1612-1616.

[3] P. Grover and A. Sahai, "Shannon meets Tesla: Wireless information and power transfer," in Proc. IEEE Int. Symp. Inf. Theory, Jun. 2010, pp. 2363-2367.

[4] R. Zhang and C. K. Ho, "MIMO broadcasting for simultaneous wireless information and power transfer," IEEE Trans. Wireless Commun., vol. 12, pp. 1989-200, May 2013.

[5] V. Sharma, U. Mukherji, V. Joseph, and S. Gupta, "Optimal energy management policies for energy harvesting sensor nodes," IEEE Trans. Wireless Commun., vol. 9, no. 4, pp. 1326-1336, Apr. 2010.

[6] C. K. Ho and R. Zhang, "Optimal energy allocation for wireless communications with energy harvesting constraints," IEEE Trans. Signal Process., vol. 60, no. 9, pp. 4808-4818, Sep. 2012.

[7] Z. Xiang and M. Tao, "Robust beamforming for wireless information and power transmission," IEEE Wireless Commun. Letters, vol. 1, pp. 372-375, Aug. 2012.

[8] L. Liu, R. Zhang, and K.-C. Chua, "Wireless information transfer with opportunistic energy harvesting," IEEE Trans. Wireless Commun., vol. 12, no. 1, pp. 288-300, Jan. 2013.

[9] L. Liu, R. Zhang, and K.-C. Chua, "Secrecy wireless information and power transfer with MISO beamforming," IEEE Trans. Signal Process. vol. 62, pp. 1850-1863, Apr. 2014.

[10] M. R. A. Khandaker and K. K. Wong, "SWIPT in MISO multicasting systems," IEEE Wireless Commun. Letters, vol. 3, no. 3, pp. 277-280, Jun. 2014

[11] M. R. A. Khandaker and K. K. Wong, "Robust secrecy beamforming in the presence of energy-harvesting eavesdroppers," IEEE Wireless Commun. Letters, vol. 4, no. 1, pp. 10-13, Feb. 2015.

[12] M. R. A. Khandaker, and K. K. Wong, "Masked beamforming in the presence of energy-harvesting eavesdroppers," IEEE Trans. Inf. Forensics and Security, vol. 10, no. 1, pp. 40-54, Jan. 2015.

[13] S. Lee, L. Liu, and R. Zhang, "Collaborative wireless energy and information transfer in interference channel," arXiv preprint arXiv:1402.6441, 2014.

[14] A. Nasir, X. Zhou, S. Durrani, and R. Kennedy, "Block-wise timeswitching energy harvesting protocol for wireless-powered AF relays," in Proc. IEEE Int. Conf. Commun., 8-12 Jun. 2015, London, UK.
[15] N. Badruddin, J. Evans, and S. Hanly, "Maximising sum rate for two interfering wireless links," in Proc. Austrialian Commun. Theory Workshop, pp. 75-81, 30 Jan.-1 Feb. 2008, Christchurch, Australia.

[16] A. Gjendemsjo, D. Gesbert, G. E. Oien, and S. G. Kiani, "Optimal power allocation and scheduling for two-cell capacity maximization," in Proc. Int. Simp. Modeling and Optimization in Mobile, Ad Hoc and Wireless Networks, pp. 1-6, 3-6 Apr. 2006, Boston, Massachusetts, USA.

[17] A. Gjendemsj, D. Gesbert, G. E. Oien, and S. G. Kiani, "Binary power control for sum rate maximization over multiple interfering links," IEEE Trans. Wireless Commun., vol. 7, no. 8, pp. 3164-3173, Aug. 2008.

[18] Q. Sun and L. Li, "Weighted sum rate maximization for downlink multiuser relay network with direct link," Wireless Per. Commun., vol. 75, no. 1, pp. 369-384, Aug. 2013.

[19] N. ul Hassan, C. Yuen, S. Saeed, and Z. Zhang, "Power control for sum rate maximization on interference channels under sum power constraint," IEEE Trans. Veh. Technol., vol. 64, no. 2, pp. 593-609, Feb. 2015.

[20] C. S. Chen and G. E. Oien, "Optimal power allocation for two-cell sum rate maximization under minimum rate constraints," in Proc. Int. Symp. Wireless Commun. Systems, pp. 396-400, 21-24 Oct. 2008, Reykjavik.

[21] J. L. Troutman, Variational calculus and optimal control: Optimization with elementary convexity, 2nd ed. New York: Springer Verlag, 1996.

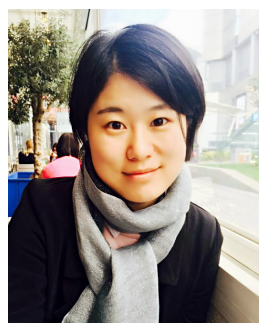

Yongxu Zhu received the B.E. degree from Nanjing University of Posts and Telecommunications, in 2010, and the M.S. degree from the Beijing University of Posts and Telecommunications and Dublin City Univeristy, in 2012 and 2013. She is currently pursuing her PhD in Wireless Communications at the Department of Electronic and Electrical Engineering, University College London, United Kingdom.

Her research interests are in the areas of Energyharvesting wireless communications, power control, Heterogeneous cellular networks, Massive MIMO,

Physical-layer security.

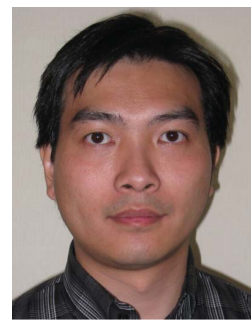

Kai-Kit Wong (M'01-SM'08) received the BEng, the MPhil, and the $\mathrm{PhD}$ degrees, all in Electrical and Electronic Engineering, from the Hong Kong University of Science and Technology, Hong Kong, in 1996, 1998, and 2001, respectively. He is Professor in Wireless Communications at the Department of Electronic and Electrical Engineering, University College London, United Kingdom.

He is Fellow of IEEE in 2016 and Fellow of IET. $\mathrm{He}$ is on the editorial board of IEEE Wireless Communications Letters, IEEE Communications Letters, IEEE ComSoc/KICS Journal of Communications and Networks, and IET Communications. He is Senior Editor for the IEEE Communications Letters and IEEE Wireless Communications Letters. He also served as Editor for IEEE Transactions on Wireless Communications from 2005-2011 and IEEE Signal Processing Letters from 2009-2012.

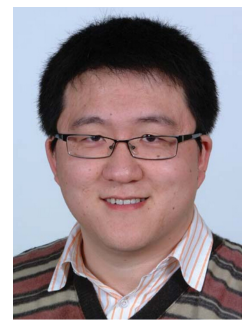

Yangyang Zhang received the B.S. and M.S. degrees in Electronics and Information Engineering from Northeastern University, Shenyang, China, in 2002 and 2004 respectively, and the Ph.D. degree in Electrical Engineering from the University of Oxford,Oxford, U.K., in 2008. He is currently taking the position of Executive Vice President in KuangChi Institute of Advanced Technology, China. His research interests include MIMO wireless communications and stochastic optimization algorithms. Dr.Zhang has been awarded more than 20 honors. Besides, he also authored and co-authored more than 30 refereed papers. 


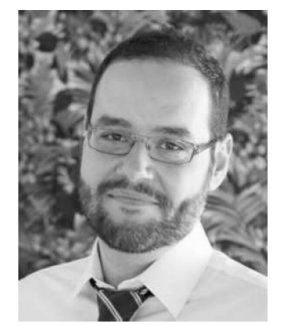

Christos Masouros (M'06-SM'14) received his Diploma in Electrical \& Computer Engineering from the University of Patras, Greece, in 2004, MSc by research and $\mathrm{PhD}$ in Electrical \& Electronic Engineering from the University of Manchester, UK in 2006 and 2009 respectively. He has previously held a Research Associate position in University of Manchester, UK and a Research Fellow position in Queens University Belfast, UK. He is currently a Lecturer in the Department of Electronic and Electrical Engineering, University College London. He holds a Royal Academy of Engineering Research Fellowship 20112016 and is the principal investigator of the EPSRC project EP/M014150/1 on large scale antenna systems. His research interests lie in the field of wireless communications and signal processing with particular focus on Green Communications, Large Scale Antenna Systems, Cognitive Radio, interference mitigation techniques for MIMO and multicarrier communications. He was awarded the Best Paper Award in IEEE GlobeCom 2015. He is an Associate Editor for IEEE Communications Letters. 